\title{
CONVERGENCE RATE OF INERTIAL PROXIMAL ALGORITHMS WITH GENERAL EXTRAPOLATION AND PROXIMAL COEFFICIENTS
}

\author{
HEDY ATTOUCH, ZAKI CHBANI, AND HASSAN RIAHI
}

\begin{abstract}
In a Hilbert space setting, with the aim of developing rapid methods for nonsmooth convex optimization, we analyze the convergence rate of the inertial proximal algorithms. These algorithms involve both extrapolation coefficients (including Nesterov acceleration method) and proximal coefficients in a general form. They can be interpreted as the discrete time version of inertial continuous gradient systems with general damping and time scale coefficients. Based on the proper setting of these parameters, we show the fast convergence of values and the convergence of iterates. In doing so, we provide an overview of this class of algorithms. Our study complements the previous Attouch-Cabot paper (SIOPT, 2018) by introducing into the algorithm time scaling aspects, and sheds new light on the Güler seminal papers on the convergence rate of the accelerated proximal methods for convex optimization.
\end{abstract}

Key words: Inertial proximal algorithms; general extrapolation coefficient; Lyapunov analysis; Nesterov accelerated gradient method; nonsmooth convex optimization; time rescaling.

AMS subject classification. 37N40, 46N10, 49M30, 65B99, 65K05, 65K10, 90B50, 90C25.

\section{INTRODUCTION}

Throughout the paper, $\mathcal{H}$ is a real Hilbert space endowed with the scalar product $\langle\cdot, \cdot\rangle$ and the corresponding norm $\|\cdot\|$. The function $\Phi: \mathcal{H} \rightarrow \mathbb{R} \cup\{+\infty\}$, which is to minimize, is supposed to be convex lower semicontinuous and proper, and such that $\operatorname{argmin}_{\mathcal{H}} \Phi \neq \emptyset$. We will analyze the convergence properties of the Inertial Proximal algorithm

$$
(\mathrm{IP})_{\alpha_{k}, \beta_{k}}\left\{\begin{array}{l}
y_{k}=x_{k}+\alpha_{k}\left(x_{k}-x_{k-1}\right) \\
x_{k+1}=\operatorname{prox}_{\beta_{k} \Phi}\left(y_{k}\right),
\end{array}\right.
$$

where the sequences $\left(\alpha_{k}\right)$ and $\left(\beta_{k}\right)$ play the role of parameters. As a standing assumption, we assume that, for every $k \geq 1, \alpha_{k}$ is non-negative and $\beta_{k}$ is positive. For a judicious choice of $\alpha_{k}$ and $\beta_{k}$, we will obtain fast convergence of the values and convergence of the iterates. In doing so, we will extend previous studies to the case of inertial proximal methods with general coefficients, including large proximal steps $\beta_{k}$. We recall that, for $\beta>0$, the proximal mapping $\operatorname{prox}_{\beta \Phi}: \mathcal{H} \rightarrow \mathcal{H}$ is defined by: for every $x \in \mathcal{H}$,

$$
\operatorname{prox}_{\beta \Phi}(x)=\operatorname{argmin}_{\xi \in \mathcal{H}}\left\{\beta \Phi(\xi)+\frac{1}{2}\|x-\xi\|^{2}\right\} .
$$

One can consult $[15,28,29,32]$, for a recent account on the proximal methods, that play a central role in nonsmooth optimization as a basic block of many splitting algorithms. Proximal methods have a close relationship with gradient-type continuous evolution systems, from which they can be derived by implicit discretization. As such, their retain their convergence properties (see [30] for first-order evolution systems). Precisely, to guide our study, when $\Phi$ is continuously differentiable, we will use the link between the (IP) ${ }_{\alpha_{k}, \beta_{k}}$ proximal algorithm and the continuous second-order evolution equation

$$
(\mathrm{IGS})_{\gamma, \beta} \quad \ddot{x}(t)+\gamma(t) \dot{x}(t)+\beta(t) \nabla \Phi(x(t))=0 .
$$

In (IGS) ${ }_{\gamma, \beta}, \quad \gamma(t)$ is a positive damping coefficient, and $\beta(t)$ is a time scale coefficient. The inertial proximal algorithm (IP) $)_{\alpha_{k}, \beta_{k}}$ can be interpreted as an implicit discretization of (IGS) ${ }_{\gamma, \beta}$, which gives the following dynamic interpretation of the parameters $\left(\alpha_{k}\right)$ and $\left(\beta_{k}\right)$ :

- The sequence $\left(\alpha_{k}\right)$ of non-negative extrapolation coefficients captures the inertial effect. The property $\alpha_{k} \rightarrow 1$, which plays a central role in the Nesterov acceleration method, is associated with the vanishing damping property $\gamma(t) \rightarrow 0$, as $t \rightarrow+\infty$.

Date: February 14, 2019. 
- The sequence $\left(\beta_{k}\right)$ of positive proximal coefficients takes into account the temporal scale effects. The coefficient $\beta_{k}$ in (IP $)_{\alpha_{k}, \beta_{k}}$ is the discrete temporal version of $\beta(t)$. The fast time parametrization of the orbits (respectively iterates) results from the property $\beta(t) \rightarrow+\infty$ as $t \rightarrow+\infty$ (respectively $\beta_{k} \rightarrow+\infty$ as $k \rightarrow+\infty)$. It plays a key role in obtaining fast optimization properties.

1.1. Presentation of the results. Historical aspects. Recall two important instances of (IP) $\alpha_{k}, \beta_{k}$ already studied, and which constitute special cases of our study:

1. The case $\beta_{k} \equiv \beta>0$ where the proximal parameter is fixed, and $\alpha_{k}$ is general, is written as follows

$$
(\mathrm{IP})_{\alpha_{k}, \beta}\left\{\begin{array}{l}
y_{k}=x_{k}+\alpha_{k}\left(x_{k}-x_{k-1}\right) \\
x_{k+1}=\operatorname{prox}_{\beta \Phi}\left(y_{k}\right) .
\end{array}\right.
$$

It was analyzed by Attouch-Cabot [5], then by Attouch-Cabot-Chbani-Riahi [6] in the presence of perturbations, errors. The following result gives the convergence rate of values in this case. It is expressed with the help of the sequence $\left(t_{k}\right)$ that is related to $\left(\alpha_{k}\right)$ by the formula $t_{k}:=1+\sum_{i=k}^{+\infty} \prod_{j=k}^{i} \alpha_{j}$. The inverse mapping takes the simpler form $\alpha_{k}=\frac{t_{k}-1}{t_{k+1}}$.

Theorem 1.1 (Attouch-Cabot [5]). Suppose that the sequence $\left(\alpha_{k}\right)$ satisfies the following properties $\left(K_{0}\right)$ and $\left(K_{1}\right)$.

$$
\begin{aligned}
& \left(K_{0}\right) \quad \forall k \geq 1, \quad \sum_{i=k}^{+\infty} \prod_{j=k}^{i} \alpha_{j}<+\infty, \\
& \left(K_{1}\right) \quad \forall k \geq 1, \quad t_{k+1}^{2}-t_{k}^{2} \leq t_{k+1} .
\end{aligned}
$$

Then, for any sequence $\left(x_{k}\right)$ generated by the algorithm $(\mathrm{IP})_{\alpha_{k}, \beta}$

$$
\Phi\left(x_{k}\right)-\min _{\mathcal{H}} \Phi=\mathcal{O}\left(\frac{1}{t_{k}^{2}}\right) \quad \text { as } k \rightarrow+\infty .
$$

This result comes as a result of a rich history. In a seminal paper [26], Nesterov proposed the extrapolation coefficient $\alpha_{k}=\frac{t_{k}-1}{t_{k+1}}$ with $t_{1}=1$ and $t_{k+1}=\frac{\sqrt{4 t_{k}^{2}+1}+1}{2}$. This choice corresponds to taking equality in $\left(K_{1}\right)$, and leads to an increasing sequence $\left(\alpha_{k}\right)$ which behaves like $1-\frac{3}{k}$ as $k \rightarrow+\infty$. Then $t_{k}$ is of order $k$, and the corresponding convergence rate for values is $\mathcal{O}\left(\frac{1}{k^{2}}\right)$. This result has been extended by Beck-Teboulle to structured convex minimization problems in [16], that's the FISTA inertial proximal-gradient algorithm. Since the introduction of Nesterov's scheme, much progress has been made in the development of first-order accelerated methods, to name a few $[15,16,18,19,22,23,24,28,32,37,35]$. Recently, a special attention has been devoted to the case $\alpha_{k}=1-\frac{\alpha}{k}$, where $\alpha>0$ (or an equivalent expression), see [8,11, 18, 37]. Given $x_{0}, x_{1} \in \mathcal{H}$, for $k \geq 1$ the algorithm writes

$$
\left\{\begin{aligned}
y_{k} & =x_{k}+\left(1-\frac{\alpha}{k}\right)\left(x_{k}-x_{k-1}\right) \\
x_{k+1} & =\operatorname{prox}_{\beta \Phi}\left(y_{k}\right) .
\end{aligned}\right.
$$

For $\alpha=3$ we recover a first-order approximation of the original choice of Nesterov. The great novelty of the algorithm (3) is that, while keeping the same computational complexity as in the case $\alpha=3$, taking $\alpha>3$ offers many advantages. First, it ensures the convergence of the sequences $\left(x_{k}\right)$, as proved by Chambolle-Dossal [18], see [8] for complementary results. Let us recall that the convergence of the sequences generated by FISTA has not been established so far. This is a puzzling question in the study of numerical optimization methods. Second, as proved by Attouch-Peypouquet in [11], it provides the better convergence rate of values

$$
\Phi\left(x_{k}\right)-\min _{\mathcal{H}} \Phi=o\left(\frac{1}{k^{2}}\right) \quad \text { as } k \rightarrow+\infty .
$$

When taking $\alpha_{k}=1-\frac{\alpha}{k}, \alpha=3$ appears as a critical value. The subcritical case $\alpha<3$ has been recently considered by Apidopoulos-Aujol-Dossal [3] and Attouch-Chbani-Riahi [9] with the convergence rate of values $\mathcal{O}\left(\frac{1}{k^{\frac{2 \alpha}{3}}}\right)$. Theorem 1.1 allows to cover all these situations (except the subcritical case), and many others including the case $\alpha_{k}=1-\frac{c}{k^{r}}$ with $0 \leq r \leq 1$.

2. The case $\beta_{k}$ general, and $\alpha_{k}=1-\frac{\alpha}{k}$, is written as follows

$$
(\mathrm{IP})_{\alpha, \beta_{k}}\left\{\begin{array}{l}
y_{k}=x_{k}+\left(1-\frac{\alpha}{k}\right)\left(x_{k}-x_{k-1}\right) \\
x_{k+1}=\operatorname{prox}_{\beta_{k} \Phi}\left(y_{k}\right)
\end{array}\right.
$$

The following result gives the convergence rate of values for the algorithm (4). It was obtained by the authors in $[7$, Theorem 7.1]. 
Theorem 1.2 (Attouch-Chbani-Riahi [7]). Take $\alpha \geq 1$. Suppose that the sequence $\left(\beta_{k}\right)$ satisfies the growth condition: there exists $k_{1} \in \mathbb{N}$ such that for all $k \geq k_{1}$

$$
\left(H_{\beta}\right) \quad \beta_{k+1} \leq \frac{k^{2}}{(k+1)(k+2-\alpha)} \beta_{k} .
$$

Then, for any sequence $\left(x_{k}\right)$ generated by the algorithm $(\mathrm{IP})_{\alpha, \beta_{k}}$, we have

$$
\Phi\left(x_{k}\right)-\min _{\mathcal{H}} \Phi=\mathcal{O}\left(\frac{1}{k^{2} \beta_{k}}\right) \quad \text { as } k \rightarrow+\infty .
$$

In this result, the introduction of the scaling factor $\beta_{k}$ permits to pass from the convergence rate $\mathcal{O}\left(\frac{1}{k^{2}}\right)$ to $\mathcal{O}\left(\frac{1}{k^{2} \beta_{k}}\right)$. When taking $\beta_{k}=k^{\delta}$, condition $\left(H_{\beta}\right)$ is satisfied for $\delta<\alpha-3$. In this case, for a given $\delta>0$, by taking $\alpha$ large enough, namely $\alpha>3+\delta$, we can improve the convergence rate of values from $\mathcal{O}\left(\frac{1}{k^{2}}\right)$ to $\mathcal{O}\left(\frac{1}{k^{2+\delta}}\right)$ This is a clear acceleration effect.

In Theorem 2.3 we will prove the following result, which includes the two studies above (it is formulated below in a condensed form).

Theorem 1.3. Suppose that the sequence $\left(\alpha_{k}\right)$ satisfies $\left(K_{0}\right)$. Let $\left(t_{k}\right)$ be the sequence defined by $\alpha_{k}=\frac{t_{k}-1}{t_{k+1}}$. Suppose that the sequences $\left(\alpha_{k}\right)$ and $\left(\beta_{k}\right)$ satisfy jointly

$$
\left(K_{1, \alpha_{k}, \beta_{k}}\right) \quad t_{k+1}^{2} \beta_{k}-t_{k}^{2} \beta_{k-1}-t_{k+1} \beta_{k} \leq 0 \quad \text { for every } k \geq 1 .
$$

Let $\left(x_{k}\right)$ be a sequence generated by algorithm $(\mathrm{IP})_{\alpha_{k}, \beta_{k}}$. Then,

$$
\Phi\left(x_{k}\right)-\min _{\mathcal{H}} \Phi=\mathcal{O}\left(\frac{1}{t_{k}^{2} \beta_{k-1}}\right) \quad \text { as } k \rightarrow+\infty .
$$

This result permits to better understand the joint tuning of the extrapolation parameter $\alpha_{k}$ and the scaling parameter $\beta_{k}$ in $(\mathrm{IP})_{\alpha_{k}, \beta_{k}}$ which provides fast convergence of the values. Our study includes Güler's accelerated proximal algorithm, and provides new insight on this result. In Güler's algorithm, the damping coefficient is written $\alpha_{k}=g_{k}\left(\frac{1}{g_{k-1}}-1\right)$, which allows to formulate the condition $\left(K_{1, \alpha_{k}, \beta_{k}}\right)$ in a simpler form, directly on the data. Based on this appraoch, and considering various temporal discretizations, our dynamic approach with general damping and scaling coefficients provides a family of inertial proximal algorithms with fast convergence properties.

\subsection{Link with inertial gradient systems and time scaling.}

1.2.1. Link with inertial gradient systems. First verify that the inertial proximal algorithm (IP) $)_{\alpha_{k}, \beta_{k}}$ can be interpreted as a discretized version in time of the damped inertial dynamic

$$
(\mathrm{IGS})_{\gamma, \beta} \quad \ddot{x}(t)+\gamma(t) \dot{x}(t)+\beta(t) \nabla \Phi(x(t))=0 .
$$

Take a fixed time step $h>0$, and set $\tau_{k}=k h, x_{k}=x\left(\tau_{k}\right)$. Implicit discretization of $(\text { IGS })_{\gamma, \beta}$ with centered second-order variation, gives

$$
\frac{1}{h^{2}}\left(x_{k+1}-2 x_{k}+x_{k-1}\right)+\frac{\gamma(k h)}{h}\left(x_{k}-x_{k-1}\right)+\beta(k h) \nabla \Phi\left(x_{k+1}\right)=0 .
$$

Hence, $x_{k+1}+h^{2} \beta(k h) \nabla \Phi\left(x_{k+1}\right)=x_{k}+(1-h \gamma(k h))\left(x_{k}-x_{k-1}\right)$, which gives the algorithm (IP) $\alpha_{k}, \beta_{k}$

$$
\left\{\begin{aligned}
y_{k} & =x_{k}+(1-h \gamma(k h))\left(x_{k}-x_{k-1}\right) \\
x_{k+1} & =\operatorname{prox}_{h^{2} \beta(k h) \Phi}\left(y_{k}\right)
\end{aligned}\right.
$$

with extrapolation coefficient $\alpha_{k}=1-h \gamma(k h)$, and prox. parameter $h^{2} \beta(k h)$.

1.2.2. From Polyak heavy ball to vanishing damping. Take $\beta(\cdot) \equiv 1$ and discuss the role of the time-dependent parameter $\gamma(t)$ in the continuous dynamic (IGS) $)_{\gamma, 1}$. In the context of mechanics, $\gamma(t)$ is a viscous friction coefficient. Taking $\gamma(t)$ positive causes the dissipation of the global energy of the system. This plays a central role in its stabilization and optimization properties. The introduction of inertial dynamics (second-order in time) in optimization comes with B. Polyak [31] who, in 1964, introduced the so-called heavy ball with friction method, which corresponds to a constant friction coefficient $\gamma(t) \equiv \gamma_{0}>0$ in (IGS) $\gamma, 1$. It turns out that in this model, the friction is important and neutralizes the inertial effect, which prevents obtaining fast optimization methods. Indeed, taking the damping parameter $\gamma(t)$ which tends to zero as $t \rightarrow+\infty$ (vanishing damping) is a key property for obtaining fast optimization methods. A decisive step in this direction was obtained by $\mathrm{Su}$, Boyd, and Candès [37], who showed that the Nesterov acceleration method can be obtained as a discretization 
of the dynamical system (IGS) ${ }_{\gamma, 1}$ with $\gamma(t)=\frac{3}{t}$. Recently, Attouch-Chbani-Peypouquet-Redont [8] and May [25] showed convergence of the trajectories of the (IGS) $\gamma$ system with $\gamma(t)=\frac{\alpha}{t}$ and $\alpha>3$

$$
(\mathrm{AVD})_{\alpha} \quad \ddot{x}(t)+\frac{\alpha}{t} \dot{x}(t)+\nabla \Phi(x(t))=0 .
$$

They also obtained the improved convergence rate $\Phi(x(t))-\min _{\mathcal{H}} \Phi=o\left(\frac{1}{t^{2}}\right)$ as $t \rightarrow+\infty$. On the basis of the correspondence $\alpha_{k}=1-h \gamma_{k}$ (where $h$ is the discrete time step, and $\gamma_{k}=\gamma(k h)$ ), the discrete version of the vanishing damping property is that the sequence $\left(\alpha_{k}\right)$ tends to one from below as $k \rightarrow+\infty$. This makes the link with the historical aspects and the recent developments based on Nesterov acceleration method described in the previous section. For recent developments concerning the rich relations between inertial gradient systems with time-dependent friction and the inertial proximal-based algorithms, one can consult [2], [5], [7], [8], [14], [17], [37], [36].

1.2.3. Link with time scaling. The time-dependent parameter $\beta(t)$ comes from the time scaling of the dynamic. Let's illustrate it in the following model situation. Start from the (AVD) $)_{\alpha}$ system with $\gamma(t)=\frac{\alpha}{t}$ and $\alpha \geq 3$. Given a trajectory $x(\cdot)$ of $(\text { AVD })_{\alpha}$, we know that (see [4], [8], [37])

$$
\Phi(x(t))-\min _{\mathcal{H}} \Phi=\mathcal{O}\left(\frac{1}{t^{2}}\right)
$$

Let's make the following change of time variable in $(\mathrm{AVD})_{\alpha}: t=s^{p}$, where $p$ is a positive parameter. Set $y(s):=x\left(s^{p}\right)$. By the derivation chain rule, we have

$$
\ddot{y}(s)+\frac{\alpha_{p}}{s} \dot{y}(s)+p^{2} s^{2(p-1)} \nabla \Phi(y(s))=0,
$$

where $\alpha_{p}=1+(\alpha-1) p$. The convergence rate of values becomes

$$
\Phi(y(s))-\min _{\mathcal{H}} \Phi=\mathcal{O}\left(\frac{1}{s^{2 p}}\right) .
$$

For $p>1$, we have $\alpha_{p}>\alpha$, so the damping parameters for (8) are similar to those of (AVD) $\alpha$. The only major difference is the coefficient $s^{2(p-1)}$ in front of $\nabla \Phi(y(s))$, which explodes when $s \rightarrow+\infty$. From (9) we observe that the convergence rate of values can be made arbitrarily fast (in the scale of powers of $\frac{1}{s}$ ) with $p$ large. The physical intuition is clear. Fast optimization is associated with the fast parameterization of the trajectories of the $(\mathrm{AVD})_{\alpha}$ system. Our goal is to transpose these results to the discrete case, taking advantage of the fact that proximal algorithms usually inherit the properties of the continuous dynamics from which they come.

1.2.4. Geometrical damping. The optimization properties of the continuous dynamic (IGS) $\gamma, \beta$ come from its viscous damping term, which makes the system stabilize asymptotically. According to this mechanical and control point of view, it is natural to consider other types of damping. An important case is the damping driven by the Hessian which contains a rich geometric information, as considered in [2], [10], [12]. The temporal discretization of this dynamic gives the Ravine method initially introduced by Gelfand, Tsetlin 1961, then developed by B. Polyak. The exact link was obtained in [36] using high-resolution. Another interesting damping is the dry friction, taken account in an optimization framework in [1], and which gives finite time stabilization. Developing our Lyapunov analysis in these situations is an interesting subject for further studies.

1.3. Organization of the paper. Based on Lyapunov analysis, our main convergence result is established in Section 2. Depending on the behavior of the sequences $\left(\alpha_{k}\right)$ and $\left(\beta_{k}\right)$, we give the convergence rate of the values of the sequences $\left(x_{k}\right)$ generated by algorithm $(\mathrm{IP})_{\alpha_{k}, \beta_{k}}$. In Section 3, we analyze the convergence rate of the velocities. In Section 4 , we show how to pass from $\mathcal{O}$ to $o$ estimates. In Section 5 , we give general conditions on the sequences $\left(\alpha_{k}\right)$ and $\left(\beta_{k}\right)$ which guarantee the weak convergence of the iterates. In Section 6 , we study the link with the accelerated proximal algorithm of Güler. In Section 7 we study a parametrized family of rescaled proximal algorithms which extends the previous study. In Section 8, we study the stability with respect to perturbations. The paper is completed by some auxiliary technical lemmas contained in the Appendix.

\section{Convergence Rate of the values}

The algorithm $(\mathrm{IP})_{\alpha_{k}, \beta_{k}}$

$$
(\mathrm{IP})_{\alpha_{k}, \beta_{k}}\left\{\begin{array}{l}
y_{k}=x_{k}+\alpha_{k}\left(x_{k}-x_{k-1}\right) \\
x_{k+1}=\operatorname{prox}_{\beta_{k} \Phi}\left(y_{k}\right)
\end{array}\right.
$$

can be equivalently formulated as

$$
x_{k+1}-2 x_{k}+x_{k-1}+\left(1-\alpha_{k}\right)\left(x_{k}-x_{k-1}\right)+\beta_{k} \partial \Phi\left(x_{k+1}\right) \ni 0 .
$$


This formulation can also be obtained from the implicit discretization of the inertial dynamic (IGS) ${ }_{\gamma, \beta}$, see (5). Following [4], let us introduce the sequence $\left(t_{k}\right)$ that plays a central role in the analysis of algorithm (IP) $)_{\alpha_{k}, \beta_{k}}$.

2.1. The sequence $\left(t_{k}\right)$. Given $k \geq 1$, assume that $\sum_{i=k}^{+\infty} \prod_{j=k}^{i} \alpha_{j}<+\infty$ and set $t_{k}=1+\sum_{i=k}^{+\infty} \prod_{j=k}^{i} \alpha_{j}$. We use the convention $\prod_{j=k}^{k-1} \alpha_{j}=1$, which gives the compact form of $t_{k}$

$$
t_{k}=\sum_{i=k-1}^{+\infty} \prod_{j=k}^{i} \alpha_{j}
$$

If the series defining $t_{k+1}$ is convergent, the series defining $t_{k}$ is also convergent, and we have

$$
1+\alpha_{k} t_{k+1}=1+\alpha_{k}\left(\sum_{i=k}^{+\infty} \prod_{j=k+1}^{i} \alpha_{j}\right)=1+\sum_{i=k}^{+\infty} \prod_{j=k}^{i} \alpha_{j}=t_{k} .
$$

Conversely, if $\alpha_{k} \neq 0$ and if $t_{k}$ is well-defined, then the series defining $t_{k+1}$ is convergent and the above equalities hold true. From now on, we assume that

$$
\sum_{i=k}^{+\infty} \prod_{j=k}^{i} \alpha_{j}<+\infty \quad \text { for every } k \geq 1 .
$$

Let us summarize the above results.

Lemma 2.1. Assume that the non-negative sequence $\left(\alpha_{k}\right)$ satisfies $\left(K_{0}\right)$. Then the sequence $\left(t_{k}\right)$ is well defined and satisfies for every $k \geq 1$

$$
1+\alpha_{k} t_{k+1}=t_{k} .
$$

The sequence $\left(t_{k}\right)$ allows us to formulate the conditions on the sequence $\left(\alpha_{k}\right)$ in a dense form. Although the direct formulation (12) of $\left(t_{k}\right)$ in terms of $\left(\alpha_{k}\right)$ is complicated, the inverse formulation is simple: $\alpha_{k}=\frac{t_{k}-1}{t_{k+1}}$.

2.2. Convergence rates of the values: Lyapunov analysis. Let us introduce the ingredients that will serve for the Lyapunov analysis. For $k \geq 1$, the global energy $W_{k}$ is defined by

$$
W_{k}:=\beta_{k-1}\left(\Phi\left(x_{k}\right)-\min _{\mathcal{H}} \Phi\right)+\frac{1}{2}\left\|x_{k}-x_{k-1}\right\|^{2} .
$$

Its importance is due to the fact that, for damped inertial gradient systems, it is the global energy that decreases. Given $x^{*} \in \operatorname{argmin} \Phi$, the anchor sequence $\left(h_{k}\right)$ is defined by: for $k \geq 1$

$$
h_{k}:=\frac{1}{2}\left\|x_{k}-x^{*}\right\|^{2} .
$$

Let us define the sequence $\left(\mathcal{E}_{k}\right)$ by: for every $k \geq 1$

$$
\mathcal{E}_{k}:=t_{k}^{2} \beta_{k-1}\left(\Phi\left(x_{k}\right)-\min _{\mathcal{H}} \Phi\right)+\frac{1}{2}\left\|x_{k-1}+t_{k}\left(x_{k}-x_{k-1}\right)-x^{*}\right\|^{2} .
$$

Note that

$$
\mathcal{E}_{k}=t_{k}^{2} W_{k}+\frac{1}{2}\left\|x_{k-1}-x^{*}\right\|^{2}+t_{k}\left\langle x_{k}-x_{k-1}, x_{k-1}-x^{*}\right\rangle .
$$

This formulation shows $\mathcal{E}_{k}$ as a weighted sum of the global energy, the anchor function, and the discrete time derivative of the anchor function. For our next calculation, we will use the formulation (16), which, as a main advantage, involves only non-negative terms. The following result shows that the sequence $\left(\mathcal{E}_{k}\right)$ is non-increasing, under the condition $\left(K_{1, \alpha_{k}, \beta_{k}}\right)$, that involves the two sequences $\left(\alpha_{k}\right)$, and $\left(\beta_{k}\right)$ together.

Proposition 2.2. Assume that the non-negative sequence $\left(\alpha_{k}\right)$ satisfies $\left(K_{0}\right)$. Let $\left(x_{k}\right)$ be a sequence generated by algorithm $(\mathrm{IP})_{\alpha_{k}, \beta_{k}}$, and let $\left(\mathcal{E}_{k}\right)$ be the sequence defined by (16). Then we have: for every $k \geq 1$

$$
\mathcal{E}_{k+1}-\mathcal{E}_{k} \leq\left(t_{k+1}^{2} \beta_{k}-t_{k}^{2} \beta_{k-1}-t_{k+1} \beta_{k}\right)\left(\Phi\left(x_{k}\right)-\min _{\mathcal{H}} \Phi\right) .
$$

Under the assumption

$\left(K_{1, \alpha_{k}, \beta_{k}}\right)$

$$
t_{k+1}^{2} \beta_{k}-t_{k}^{2} \beta_{k-1}-t_{k+1} \beta_{k} \leq 0 \quad \text { for every } k \geq 1,
$$

then the sequence $\left(\mathcal{E}_{k}\right)$ is non-increasing. 
Proof. By definition of the proximal operator, the iteration at step $k$ of the algorithm (IP) $\alpha_{k}, \beta_{k}$ writes

$$
\frac{1}{\beta_{k}}\left(y_{k}-x_{k+1}\right) \in \partial \Phi\left(x_{k+1}\right) .
$$

Equivalently, we have the following subdifferential inequalities: for any $x \in \mathcal{H}$

$$
\Phi(x) \geq \Phi\left(x_{k+1}\right)+\frac{1}{\beta_{k}}\left\langle x-x_{k+1}, y_{k}-x_{k+1}\right\rangle .
$$

Let us write successively inequality (18) at $x=x_{k}$ and $x=x^{*} \in \operatorname{argmin} \Phi$. We obtain the two inequalities

$$
\begin{aligned}
& \Phi\left(x_{k}\right) \geq \Phi\left(x_{k+1}\right)+\frac{1}{\beta_{k}}\left\langle x_{k}-x_{k+1}, y_{k}-x_{k+1}\right\rangle, \\
& \Phi\left(x^{*}\right) \geq \Phi\left(x_{k+1}\right)+\frac{1}{\beta_{k}}\left\langle x^{*}-x_{k+1}, y_{k}-x_{k+1}\right\rangle .
\end{aligned}
$$

Using $x_{k}-x_{k+1}=x_{k}-y_{k}+y_{k}-x_{k+1}$ in (19) and $x^{*}-x_{k+1}=x^{*}-y_{k}+y_{k}-x_{k+1}$ in (20) we obtain

$$
\begin{aligned}
& \Phi\left(x_{k}\right) \geq \Phi\left(x_{k+1}\right)+\frac{1}{\beta_{k}}\left\langle x_{k}-y_{k}, y_{k}-x_{k+1}\right\rangle+\frac{1}{\beta_{k}}\left\|y_{k}-x_{k+1}\right\|^{2}, \\
& \Phi\left(x^{*}\right) \geq \Phi\left(x_{k+1}\right)+\frac{1}{\beta_{k}}\left\langle x^{*}-y_{k}, y_{k}-x_{k+1}\right\rangle+\frac{1}{\beta_{k}}\left\|y_{k}-x_{k+1}\right\|^{2} .
\end{aligned}
$$

Multiplying (21) by $t_{k+1}-1 \geq 0$, then adding $(22)$, we derive that

$$
\begin{aligned}
\left(t_{k+1}-1\right)\left(\Phi\left(x_{k}\right)-\Phi\left(x^{*}\right)\right) & \geq t_{k+1}\left(\Phi\left(x_{k+1}\right)-\Phi\left(x^{*}\right)\right)+\frac{1}{\beta_{k}}\left\langle x_{k+1}-y_{k},\left(t_{k+1}-1\right)\left(y_{k}-x_{k}\right)+y_{k}-x^{*}\right\rangle \\
& +\frac{t_{k+1}}{\beta_{k}}\left\|y_{k}-x_{k+1}\right\|^{2} .
\end{aligned}
$$

Observe that

$$
\begin{aligned}
\left(t_{k+1}-1\right)\left(y_{k}-x_{k}\right)+y_{k} & =t_{k+1} y_{k}-\left(t_{k+1}-1\right) x_{k} \\
& =x_{k}+t_{k+1} \alpha_{k}\left(x_{k}-x_{k-1}\right) \\
& =x_{k-1}+\left(1+t_{k+1} \alpha_{k}\right)\left(x_{k}-x_{k-1}\right) \\
& =x_{k-1}+t_{k}\left(x_{k}-x_{k-1}\right) \quad \text { in view of }(13) .
\end{aligned}
$$

Setting $z_{k}=x_{k-1}+t_{k}\left(x_{k}-x_{k-1}\right)$, we then deduce from (23) that

$$
\begin{aligned}
\left(t_{k+1}-1\right)\left(\Phi\left(x_{k}\right)-\Phi\left(x^{*}\right)\right) & \geq t_{k+1}\left(\Phi\left(x_{k+1}\right)-\Phi\left(x^{*}\right)\right) \\
& +\frac{1}{\beta_{k}}\left\langle x_{k+1}-y_{k}, z_{k}-x^{*}\right\rangle+\frac{t_{k+1}}{\beta_{k}}\left\|y_{k}-x_{k+1}\right\|^{2}
\end{aligned}
$$

Equivalently, after multiplication by $\beta_{k}$

$$
\begin{aligned}
\beta_{k}\left(t_{k+1}-1\right)\left(\Phi\left(x_{k}\right)-\Phi\left(x^{*}\right)\right) & \geq \beta_{k} t_{k+1}\left(\Phi\left(x_{k+1}\right)-\Phi\left(x^{*}\right)\right) \\
& +\left\langle x_{k+1}-y_{k}, z_{k}-x^{*}\right\rangle+t_{k+1}\left\|y_{k}-x_{k+1}\right\|^{2} .
\end{aligned}
$$

To write (24) in a recursive form, observe that

$$
\begin{aligned}
z_{k+1}-z_{k} & =x_{k}+t_{k+1}\left(x_{k+1}-x_{k}\right)-x_{k-1}-t_{k}\left(x_{k}-x_{k-1}\right) \\
& =t_{k+1}\left(x_{k+1}-x_{k}\right)-\left(t_{k}-1\right)\left(x_{k}-x_{k-1}\right) \\
& =t_{k+1}\left(x_{k+1}-x_{k}-\alpha_{k}\left(x_{k}-x_{k-1}\right)\right) \quad \text { in view of }(13) \\
& =t_{k+1}\left(x_{k+1}-y_{k}\right) .
\end{aligned}
$$

It ensues that

$$
\left\|z_{k+1}-x^{*}\right\|^{2}=\left\|z_{k}-x^{*}\right\|^{2}+2 t_{k+1}\left\langle x_{k+1}-y_{k}, z_{k}-x^{*}\right\rangle+t_{k+1}^{2}\left\|x_{k+1}-y_{k}\right\|^{2},
$$

which gives

$$
\left\langle x_{k+1}-y_{k}, z_{k}-x^{*}\right\rangle=\frac{1}{2 t_{k+1}}\left(\left\|z_{k+1}-x^{*}\right\|^{2}-\left\|z_{k}-x^{*}\right\|^{2}\right)-\frac{t_{k+1}}{2}\left\|x_{k+1}-y_{k}\right\|^{2} .
$$

Using this equality in (24), we find

$$
\begin{aligned}
\beta_{k}\left(t_{k+1}-1\right)\left(\Phi\left(x_{k}\right)-\Phi\left(x^{*}\right)\right) & \geq \beta_{k} t_{k+1}\left(\Phi\left(x_{k+1}\right)-\Phi\left(x^{*}\right)\right)+\frac{t_{k+1}}{2}\left\|y_{k}-x_{k+1}\right\|^{2} \\
& +\frac{1}{2 t_{k+1}}\left(\left\|z_{k+1}-x^{*}\right\|^{2}-\left\|z_{k}-x^{*}\right\|^{2}\right) .
\end{aligned}
$$


After multiplication by $t_{k+1}$, and neglecting the non-negative term $\frac{t_{k+1}}{2}\left\|y_{k}-x_{k+1}\right\|^{2}$, we obtain

$$
\beta_{k}\left(t_{k+1}^{2}-t_{k+1}\right)\left(\Phi\left(x_{k}\right)-\Phi\left(x^{*}\right)\right)+\frac{1}{2}\left\|z_{k}-x^{*}\right\|^{2} . \geq \beta_{k} t_{k+1}^{2}\left(\Phi\left(x_{k+1}\right)-\Phi\left(x^{*}\right)\right)+\frac{1}{2}\left\|z_{k+1}-x^{*}\right\|^{2} .
$$

Note that the sequence $\left(\mathcal{E}_{k}\right)$ writes equivalently $\mathcal{E}_{k}=t_{k}^{2} \beta_{k-1}\left(\Phi\left(x_{k}\right)-\min _{\mathcal{H}} \Phi\right)+\frac{1}{2}\left\|z_{k}-x^{*}\right\|^{2}$. Thus, we have obtained the following inequality

$$
\mathcal{E}_{k} \geq \mathcal{E}_{k+1}+\left(t_{k}^{2} \beta_{k-1}-\beta_{k}\left(t_{k+1}^{2}-t_{k+1}\right)\right)\left(\Phi\left(x_{k}\right)-\min _{\mathcal{H}} \Phi\right) .
$$

Under condition $\left(K_{1, \beta_{k}}\right)$ we have $t_{k}^{2} \beta_{k-1}-\beta_{k}\left(t_{k+1}^{2}-t_{k+1}\right) \geq 0$. As a consequence $\mathcal{E}_{k} \geq \mathcal{E}_{k+1}$, and the sequence $\left(\mathcal{E}_{k}\right)$ is non-increasing.

As a result of Proposition 2.2, we obtain the convergence rate of values as shown below.

Theorem 2.3. Suppose that the sequence $\left(\alpha_{k}\right)$ satisfies $\left(K_{0}\right)$. Suppose that $\left(\alpha_{k}\right)$ and $\left(\beta_{k}\right)$ satisfy $\left(K_{1, \alpha_{k}, \beta_{k}}\right)$. Let $\left(x_{k}\right)$ be a sequence generated by algorithm $(\mathrm{IP})_{\alpha_{k}, \beta_{k}}$. Then we have

(i) For every $k \geq 1$,

$$
\Phi\left(x_{k}\right)-\min _{\mathcal{H}} \Phi \leq \frac{C}{t_{k}^{2} \beta_{k-1}},
$$

with $C=t_{1}^{2} \beta_{0}\left(\Phi\left(x_{1}\right)-\min _{\mathcal{H}} \Phi\right)+\frac{1}{2}\left(d\left(x_{0}, \operatorname{argmin}_{\mathcal{H}} \Phi\right)^{2}+t_{1}^{2}\left\|x_{1}-x_{0}\right\|^{2}\right)$.

(ii) Assume moreover that there exists $m<1$ such that

$\left(K_{1, \alpha_{k}, \beta_{k}}^{+}\right) \quad t_{k+1}^{2} \beta_{k}-t_{k}^{2} \beta_{k-1} \leq m t_{k+1} \beta_{k} \quad$ for every $k \geq 1$.

Then we have

$$
\sum_{k=1}^{+\infty} t_{k+1} \beta_{k}\left(\Phi\left(x_{k}\right)-\min _{\mathcal{H}} \Phi\right)<+\infty
$$

Proof. (i) From Proposition 2.2, the sequence $\left(\mathcal{E}_{k}\right)$ is non-increasing. It ensues that $\mathcal{E}_{k} \leq \mathcal{E}_{1}$ for every $k \geq 1$. Recalling the expression of $\mathcal{E}_{k}$, we deduce that

$$
\begin{aligned}
t_{k}^{2} \beta_{k-1}\left(\Phi\left(x_{k}\right)-\min _{\mathcal{H}} \Phi\right) & \leq t_{1}^{2} \beta_{0}\left[\Phi\left(x_{1}\right)-\min _{\mathcal{H}} \Phi\right]+\frac{1}{2}\left\|x_{0}-x^{*}+t_{1}\left(x_{1}-x_{0}\right)\right\|^{2} \\
& \leq t_{1}^{2} \beta_{0}\left[\Phi\left(x_{1}\right)-\min _{\mathcal{H}} \Phi\right]+\frac{1}{2}\left\|x_{0}-x^{*}\right\|^{2}+\frac{t_{1}^{2}}{2}\left\|x_{1}-x_{0}\right\|^{2}
\end{aligned}
$$

Since $x^{*}$ can be taken arbitrarily in $\operatorname{argmin}_{\mathcal{H}} \Phi$, we finally obtain

$$
t_{k}^{2} \beta_{k-1}\left(\Phi\left(x_{k}\right)-\min _{\mathcal{H}} \Phi\right) \leq C
$$

with

$$
C=t_{1}^{2} \beta_{0}\left(\Phi\left(x_{1}\right)-\min _{\mathcal{H}} \Phi\right)+\frac{1}{2}\left(d\left(x_{0}, \operatorname{argmin}_{\mathcal{H}} \Phi\right)^{2}+t_{1}^{2}\left\|x_{1}-x_{0}\right\|^{2}\right) .
$$

(ii) By summing inequality (17) from $k=1$ to $n$, we find

$$
\mathcal{E}_{n+1}+\sum_{k=1}^{n}\left(t_{k+1} \beta_{k}-t_{k+1}^{2} \beta_{k}+t_{k}^{2} \beta_{k-1}\right)\left(\Phi\left(x_{k}\right)-\min _{\mathcal{H}} \Phi\right) \leq \mathcal{E}_{1} .
$$

Since $\mathcal{E}_{n+1} \geq 0$ and since $t_{k+1}^{2} \beta_{k}-t_{k}^{2} \beta_{k-1} \leq m t_{k+1} \beta_{k}$, this implies that

$$
(1-m) \sum_{k=1}^{n} t_{k+1} \beta_{k}\left(\Phi\left(x_{k}\right)-\min _{\mathcal{H}} \Phi\right) \leq \mathcal{E}_{1} .
$$

By letting $n$ tend to infinity, we obtain

$$
\sum_{k=1}^{\infty} t_{k+1} \beta_{k}\left(\Phi\left(x_{k}\right)-\min _{\mathcal{H}} \Phi\right) \leq \frac{\mathcal{E}_{1}}{1-m},
$$

which gives the claim.

\subsection{Particular cases.}


2.3.1. Case $\beta_{k} \equiv s>0, \alpha_{k}$ general. This is the case without scaling. The algorithm (IP) $\alpha_{k}, \beta_{k}$ writes

$$
\left\{\begin{array}{l}
y_{k}=x_{k}+\alpha_{k}\left(x_{k}-x_{k-1}\right) \\
x_{k+1}=\operatorname{prox}_{s \Phi}\left(y_{k}\right)
\end{array}\right.
$$

Condition $\left(K_{1, \alpha_{k}, \beta_{k}}\right)$ becomes $t_{k+1}^{2}-t_{k}^{2}-t_{k+1} \leq 0$ for every $k \geq 1$. Under this condition, we obtain the convergence rate of values

$$
\Phi\left(x_{k}\right)-\min _{\mathcal{H}} \Phi \leq \frac{C}{t_{k}^{2}}
$$

Thus, we recover the convergence rate of values of Attouch-Cabot [4].

2.3.2. Case $\alpha_{k}=1-\frac{\alpha}{k}, \beta_{k}$ general. The algorithm (IP $)_{\alpha_{k}, \beta_{k}}$ writes

$$
\left\{\begin{array}{l}
y_{k}=x_{k}+\left(1-\frac{\alpha}{k}\right)\left(x_{k}-x_{k-1}\right) \\
x_{k+1}=\operatorname{prox}_{\beta_{k} \Phi}\left(y_{k}\right)
\end{array}\right.
$$

Then, $t_{k}=\frac{k-1}{\alpha-1}($ see $[4])$, and condition $\left(K_{1, \beta_{k}}\right)$ becomes

$$
\beta_{k+1} \leq \beta_{k} \frac{k^{2}}{(k+1)(k+2-\alpha)} .
$$

Under this condition, we obtain the convergence rate of values

$$
\Phi\left(x_{k}\right)-\min _{\mathcal{H}} \Phi \leq \frac{C}{k^{2} \beta_{k}} .
$$

Thus, we recover the result of Attouch-Chbani-Riahi [7, section 7].

\section{Convergence Rate of the velocities}

Let $\left(x_{k}\right)$ be a sequence generated by the algorithm $(\mathrm{IP})_{\alpha_{k}, \beta_{k}}$. We will analyze the convergence rate to zero of the velocity $\left\|x_{k}-x_{k-1}\right\|$. Our analysis is based on the global energy decay. Recall that the friction effect, and thus the dissipation of energy, is related to $\alpha_{k} \leq 1$.

Proposition 3.1. Let $\left(x_{k}\right)$ be a sequence generated by algorithm (IP) $)_{\alpha_{k}, \beta_{k}}$.

Then, for every $k \geq 1$,

$$
\beta_{k}\left(\Phi\left(x_{k}\right)-\min _{\mathcal{H}} \Phi\right)+\frac{1}{2}\left\|x_{k}-x_{k-1}\right\|^{2} \geq \beta_{k}\left(\Phi\left(x_{k+1}\right)-\mathcal{H} \Phi\right)+\frac{1}{2}\left\|x_{k+1}-x_{k}\right\|^{2}+\frac{1-\alpha_{k}^{2}}{2}\left\|x_{k}-x_{k-1}\right\|^{2} .
$$

Proof. The iteration at step $k$ of the algorithm (IP $)_{\alpha_{k}, \beta_{k}}$ writes $\frac{1}{\beta_{k}}\left(y_{k}-x_{k+1}\right) \in \partial \Phi\left(x_{k+1}\right)$, which gives:

$$
\Phi\left(x_{k}\right)-\min _{\mathcal{H}} \Phi \geq \Phi\left(x_{k+1}\right)-\min _{\mathcal{H}} \Phi+\frac{1}{\beta_{k}}\left\langle y_{k}-x_{k+1}, x_{k}-x_{k+1}\right\rangle .
$$

According to the extrapolation formula, $y_{k}=x_{k}+\alpha_{k}\left(x_{k}-x_{k-1}\right)$, and after multiplication by $\beta_{k}$ we get

$$
\begin{aligned}
\beta_{k}\left(\Phi\left(x_{k}\right)-\min _{\mathcal{H}} \Phi\right) & \geq \beta_{k}\left(\Phi\left(x_{k+1}\right)-\min _{\mathcal{H}} \Phi\right)+\left\langle x_{k}-x_{k+1}+\alpha_{k}\left(x_{k}-x_{k-1}\right), x_{k}-x_{k+1}\right\rangle \\
& =\beta_{k}\left(\Phi\left(x_{k+1}\right)-\min _{\mathcal{H}} \Phi\right)+\left\|x_{k}-x_{k+1}\right\|^{2}+\left\langle\alpha_{k}\left(x_{k}-x_{k-1}\right), x_{k}-x_{k+1}\right\rangle .
\end{aligned}
$$

By using the algebraic inequality

$$
\left|\left\langle\alpha_{k}\left(x_{k}-x_{k-1}\right), x_{k}-x_{k+1}\right\rangle\right| \leq \frac{1}{2}\left\|x_{k}-x_{k+1}\right\|^{2}+\frac{\alpha_{k}^{2}}{2}\left\|x_{k}-x_{k-1}\right\|^{2},
$$

we deduce that

$$
\beta_{k}\left(\Phi\left(x_{k}\right)-\min _{\mathcal{H}} \Phi\right) \geq \beta_{k}\left(\Phi\left(x_{k+1}\right)-\min _{\mathcal{H}} \Phi\right)+\left\|x_{k}-x_{k+1}\right\|^{2}-\frac{1}{2}\left\|x_{k}-x_{k+1}\right\|^{2}-\frac{\alpha_{k}^{2}}{2}\left\|x_{k}-x_{k-1}\right\|^{2} .
$$

Equivalently

$$
\beta_{k}\left(\Phi\left(x_{k}\right)-\min _{\mathcal{H}} \Phi\right)+\frac{1}{2}\left\|x_{k}-x_{k-1}\right\|^{2} \geq \beta_{k}\left(\Phi\left(x_{k+1}\right)-\min _{\mathcal{H}} \Phi\right)+\frac{1}{2}\left\|x_{k+1}-x_{k}\right\|^{2}+\frac{1-\alpha_{k}^{2}}{2}\left\|x_{k}-x_{k-1}\right\|^{2},
$$

which gives the claim.

Theorem 3.2. Suppose that $\left(\alpha_{k}\right)$ and $\left(\beta_{k}\right)$ satisfy $\left(K_{1, \alpha_{k}, \beta_{k}}^{+}\right)$. Then, we have

$$
\sum_{k=1}^{+\infty} t_{k}\left\|x_{k}-x_{k-1}\right\|^{2}<+\infty
$$


Proof. Consider, for $k \geq 1$, the global energy $W_{k}$, where we set $m=\min _{\mathcal{H}} \Phi$ :

$$
W_{k}:=\beta_{k-1}\left(\Phi\left(x_{k}\right)-m\right)+\frac{1}{2}\left\|x_{k}-x_{k-1}\right\|^{2} .
$$

Let us multiply the inequality (27) obtained in Proposition 3.1 by $t_{k+1}^{2}$

$$
\begin{aligned}
t_{k+1}^{2}\left(\beta_{k}\left(\Phi\left(x_{k}\right)-m\right)+\frac{1}{2}\left\|x_{k}-x_{k-1}\right\|^{2}\right) & \geq t_{k+1}^{2} \beta_{k}\left(\Phi\left(x_{k+1}\right)-m\right) \\
+\frac{t_{k+1}^{2}}{2}\left\|x_{k+1}-x_{k}\right\|^{2} & +\frac{t_{k+1}^{2}\left(1-\alpha_{k}^{2}\right)}{2}\left\|x_{k}-x_{k-1}\right\|^{2} .
\end{aligned}
$$

Let us reformulate this inequality in terms of the sequence $\left(W_{k}\right)$. We have

$$
\begin{array}{r}
t_{k+1}^{2}\left(\beta_{k-1}\left(\Phi\left(x_{k}\right)-m\right)+\left(\beta_{k}-\beta_{k-1}\right)\left(\Phi\left(x_{k}\right)-m\right)+\frac{1}{2}\left\|x_{k}-x_{k-1}\right\|^{2}\right) \\
\geq t_{k+1}^{2} W_{k+1}+\frac{t_{k+1}^{2}\left(1-\alpha_{k}^{2}\right)}{2}\left\|x_{k}-x_{k-1}\right\|^{2},
\end{array}
$$

which gives

$$
t_{k+1}^{2} W_{k}+t_{k+1}^{2}\left(\beta_{k}-\beta_{k-1}\right)\left(\Phi\left(x_{k}\right)-m\right) \geq t_{k+1}^{2} W_{k+1}+\frac{t_{k+1}^{2}\left(1-\alpha_{k}^{2}\right)}{2}\left\|x_{k}-x_{k-1}\right\|^{2} .
$$

Equivalently

$$
t_{k+1}^{2}\left(W_{k+1}-W_{k}\right)+\frac{t_{k+1}^{2}\left(1-\alpha_{k}^{2}\right)}{2}\left\|x_{k}-x_{k-1}\right\|^{2} \leq t_{k+1}^{2}\left(\beta_{k}-\beta_{k-1}\right)\left(\Phi\left(x_{k}\right)-m\right) .
$$

Let us rewrite the above expression as

$$
\begin{aligned}
t_{k+1}^{2} W_{k+1}-t_{k}^{2} W_{k} & +\left(t_{k}^{2}-t_{k+1}^{2}\right) W_{k}+\frac{t_{k+1}^{2}\left(1-\alpha_{k}^{2}\right)}{2}\left\|x_{k}-x_{k-1}\right\|^{2} \\
& \leq t_{k+1}^{2}\left(\beta_{k}-\beta_{k-1}\right)\left(\Phi\left(x_{k}\right)-m\right) .
\end{aligned}
$$

Recalling the expression of $W_{k}$, we deduce that

$$
\begin{aligned}
t_{k+1}^{2} W_{k+1}-t_{k}^{2} W_{k} & +\frac{1}{2}\left[t_{k}^{2}-t_{k+1}^{2}+t_{k+1}^{2}\left(1-\alpha_{k}^{2}\right)\right]\left\|x_{k}-x_{k-1}\right\|^{2} \\
& \leq\left[\left(t_{k+1}^{2}-t_{k}^{2}\right) \beta_{k-1}+t_{k+1}^{2}\left(\beta_{k}-\beta_{k-1}\right)\right]\left(\Phi\left(x_{k}\right)-m\right) .
\end{aligned}
$$

On the one hand, by (13), and since $t_{k} \geq 1$ we have

$$
t_{k}^{2}-t_{k+1}^{2}+t_{k+1}^{2}\left(1-\alpha_{k}^{2}\right)=t_{k}^{2}-t_{k+1}^{2} \alpha_{k}^{2}=t_{k}^{2}-\left(t_{k}-1\right)^{2}=2 t_{k}-1 \geq t_{k} .
$$

On the other hand, by using condition $\left(K_{1, \alpha_{k}, \beta_{k}}^{+}\right)$we have

$$
\left(t_{k+1}^{2}-t_{k}^{2}\right) \beta_{k-1}+t_{k+1}^{2}\left(\beta_{k}-\beta_{k-1}\right)=t_{k+1}^{2} \beta_{k}-t_{k}^{2} \beta_{k-1} \leq t_{k+1} \beta_{k} .
$$

Combining the above results we obtain

$$
t_{k+1}^{2} W_{k+1}-t_{k}^{2} W_{k}+\frac{1}{2} t_{k}\left\|x_{k}-x_{k-1}\right\|^{2} \leq t_{k+1} \beta_{k}\left(\Phi\left(x_{k}\right)-m\right) .
$$

By Theorem 2.3, under the assumption $\left(K_{1, \alpha_{k}, \beta_{k}}^{+}\right)$we have

$$
\sum_{k=1}^{\infty} t_{k+1} \beta_{k}\left(\Phi\left(x_{k}\right)-m\right)<+\infty .
$$

As a consequence, by summing the above inequalities, we obtain

$$
\frac{1}{2} \sum_{k=1}^{\infty} t_{k}\left\|x_{k}-x_{k-1}\right\|^{2} \leq t_{1}^{2} W_{1}+\sum_{k=1}^{\infty} t_{k+1} \beta_{k}\left(\Phi\left(x_{k}\right)-m\right)<+\infty,
$$

which gives the claim.

Corollary 3.3. Suppose that the sequences $\left(\alpha_{k}\right)$ and $\left(\beta_{k}\right)$ satisfy $\left(K_{1, \alpha_{k}, \beta_{k}}^{+}\right)$. Suppose that $0 \leq \alpha_{k} \leq 1$ for every $k \geq 1$, and that the sequence $\left(\beta_{k}\right)$ satisfies: there exists some positive constant $C$ such that, for all $k \geq 1$

$$
\beta_{k} \leq C \beta_{k+1} .
$$

Then, for any sequence $\left(x_{k}\right)$ generated by the algorithm $(\mathrm{IP})_{\alpha_{k}, \beta_{k}}$, we have

$$
\sum_{k=1}^{+\infty} t_{k} W_{k}<+\infty \text {, where } W_{k}:=\beta_{k-1}\left(\Phi\left(x_{k}\right)-\min _{\mathcal{H}} \Phi\right)+\frac{1}{2}\left\|x_{k}-x_{k-1}\right\|^{2} .
$$


Proof. By Theorem $2.3(i i)$, under the assumption $\left(K_{1, \alpha_{k}, \beta_{k}}^{+}\right)$we have

$$
\sum_{k=1}^{+\infty} t_{k+1} \beta_{k}\left(\Phi\left(x_{k}\right)-\min _{\mathcal{H}} \Phi\right)<+\infty
$$

By assumption (30) we have $t_{k} \beta_{k-1} \leq C t_{k} \beta_{k}$. By Lemma 2.1, we have $t_{k}=1+\alpha_{k} t_{k+1}$. Since $0 \leq \alpha_{k} \leq 1$, and according to $1 \leq t_{k+1}$, this implies

$$
t_{k} \leq 1+t_{k+1} \leq 2 t_{k+1}
$$

Combining the two above inequalities, we obtain $t_{k} \beta_{k-1} \leq 2 C t_{k+1} \beta_{k}$. According to (31) this implies

$$
\sum_{k=1}^{+\infty} t_{k} \beta_{k-1}\left(\Phi\left(x_{k}\right)-\min _{\mathcal{H}} \Phi\right) \leq 2 C \sum_{k=1}^{+\infty} t_{k+1} \beta_{k}\left(\Phi\left(x_{k}\right)-\min _{\mathcal{H}} \Phi\right)<+\infty .
$$

Combining this inequality with the estimate of the velocities $\sum_{k=1}^{+\infty} t_{k}\left\|x_{k}-x_{k-1}\right\|^{2}<+\infty$, obtained in Theorem 3.2 , we obtain $\sum_{k=1}^{+\infty} t_{k} W_{k}<+\infty$.

Remark 3.4. In Corollary 3.3, the assumption on the sequence $\left(\beta_{k}\right)$ is satisfied in almost all practical situations. Clearly, it is satisfied if the sequence $\left(\beta_{k}\right)$ is non-decreasing. It may be also decreasing like $\frac{1}{k^{r}}$ for any $r \geq 0$, or $\exp (-k)$. A limiting case in which it is not satisfied is $\frac{1}{k !}$. Moreover, the assumption on the sequence $\left(\alpha_{k}\right)$ can be weakened, just assuming that it is bounded from above.

\section{From $\mathcal{O}$ to o estimates}

In line with Attouch-Peypouquet [11] and Attouch-Cabot [5], we have the following convergence rate of values, where $\mathcal{O}$ is replaced by $o$.

Theorem 4.1. Let us assume that the non-negative sequence $\left(\alpha_{k}\right)$ is bounded from above, $\left(\alpha_{k}\right)$ and $\left(\beta_{k}\right)$ satisfy the condition $\left(K_{1, \alpha_{k}, \beta_{k}}^{+}\right)$, and there exists some positive constant $C$ such that $\beta_{k} \leq C \beta_{k+1}$ for all $k \geq 1$.

Then, for any sequence $\left(x_{k}\right)$ generated by the algorithm (IP $)_{\alpha_{k}, \beta_{k}}$, we have:

i) If $\sum \frac{1}{t_{k}}=+\infty$, then

$$
\Phi\left(x_{k}\right)-\min _{\mathcal{H}} \Phi=o\left(\frac{1}{t_{k}^{2} \beta_{k}}\right) \text { and }\left\|x_{k}-x_{k-1}\right\|^{2}=o\left(\frac{1}{t_{k}^{2}}\right) .
$$

ii) If $\sum t_{k} \beta_{k-1}=+\infty, 0 \leq \alpha_{k} \leq 1$, and $\left(\beta_{k}\right)$ is non-decreasing, then

$$
\Phi\left(x_{k}\right)-\min _{\mathcal{H}} \Phi=o\left(\frac{1}{\sum_{i=1}^{k} t_{i} \beta_{i-1}}\right) \text { and }\left\|x_{k}-x_{k-1}\right\|^{2}=o\left(\frac{\beta_{k-1}}{\sum_{i=1}^{k} t_{i} \beta_{i-1}}\right) .
$$

In particular, $\Phi\left(x_{k}\right) \rightarrow \min _{\mathcal{H}} \Phi$ as $k \rightarrow+\infty$.

Proof. Let's consider the sequence of global energies $\left(W_{k}\right)$ introduced in the proof of Theorem 3.2

$$
W_{k}:=\beta_{k-1}\left(\Phi\left(x_{k}\right)-m\right)+\frac{1}{2}\left\|x_{k}-x_{k-1}\right\|^{2}
$$

By Corollary 3.3 it satisfies

$$
\sum_{k=1}^{\infty} t_{k} W_{k}<+\infty
$$

i) Returning to (29) we have

$$
t_{k+1}^{2} W_{k+1}-t_{k}^{2} W_{k}+\frac{1}{2} t_{k}\left\|x_{k}-x_{k-1}\right\|^{2} \leq t_{k+1} \beta_{k}\left(\Phi\left(x_{k}\right)-m\right) .
$$

The non-negative sequence $\left(a_{k}\right)$ with $a_{k}:=t_{k}^{2} W_{k}$ satisfies the relation

$$
a_{k+1}-a_{k} \leq \omega_{k}
$$

with $\omega_{k}=t_{k+1} \beta_{k}\left(\Phi\left(x_{k}\right)-m\right)$. According to $\sum_{k \geq 1} t_{k+1} \beta_{k}\left(\Phi\left(x_{k}\right)-m\right)<+\infty$ (see Theorem $\left.2.3(i i)\right)$ we have $\left(w_{k}\right) \in l^{1}(\mathbb{N})$. By a standard argument, we deduce that the limit of the sequence $\left(a_{k}\right)$ exists, that is

$$
\lim _{k \rightarrow+\infty} t_{k}^{2} W_{k} \quad \text { exists. }
$$

Let $c:=\lim _{k \rightarrow+\infty} t_{k}^{2} W_{k}$. Let's show that $c=0$. Let's argue by contradiction, and suppose that $c>0$. As a result, $t_{k} W_{k} \sim \frac{c}{t_{k}}$. According to $\sum_{k=1}^{\infty} t_{k} W_{k}<+\infty$, this implies $c \sum \frac{1}{t_{k}}<+\infty$, a clear contradiction with the hypothesis $\sum \frac{1}{t_{k}}=+\infty$. Hence, $c=0$. So, $\lim _{k \rightarrow+\infty} t_{k}^{2} W_{k}=0$, which gives the claim. 
ii) The argument is based on the non-increasing property of the sequence $\left(\frac{1}{\beta_{k-1}} W_{k}\right)$. Let us return to Proposition 3.1. After dividing (27) by $\beta_{k}$, we get

$$
\begin{aligned}
\left(\Phi\left(x_{k}\right)-\min _{\mathcal{H}} \Phi\right)+ & \frac{1}{2 \beta_{k}}\left\|x_{k}-x_{k-1}\right\|^{2} \geq\left(\Phi\left(x_{k+1}\right)-\min _{\mathcal{H}} \Phi\right) \\
& +\frac{1}{2 \beta_{k}}\left\|x_{k+1}-x_{k}\right\|^{2}+\frac{1-\alpha_{k}^{2}}{2 \beta_{k}}\left\|x_{k}-x_{k-1}\right\|^{2} .
\end{aligned}
$$

Let's formulate the above inequality in terms of the sequence $\left(W_{k}\right)$. We obtain

$$
\frac{1}{\beta_{k-1}} W_{k}+\left(\frac{1}{2 \beta_{k}}-\frac{1}{2 \beta_{k-1}}\right)\left\|x_{k}-x_{k-1}\right\|^{2} \geq \frac{1}{\beta_{k}} W_{k+1}+\frac{1-\alpha_{k}^{2}}{2 \beta_{k}}\left\|x_{k}-x_{k-1}\right\|^{2} .
$$

Since $0 \leq \alpha_{k} \leq 1$, and the sequence $\left(\beta_{k}\right)$ is non-decreasing, we deduce that

$$
\frac{1}{\beta_{k-1}} W_{k} \geq \frac{1}{\beta_{k}} W_{k+1} \text {. }
$$

Hence, the sequence $\left(\frac{1}{\beta_{k-1}} W_{k}\right)$ is non-increasing. Let us rewrite the estimate $\sum_{k=1}^{\infty} t_{k} W_{k}<+\infty$. as

$$
\sum_{k=1}^{\infty} t_{k} \beta_{k-1}\left(\frac{1}{\beta_{k-1}} W_{k}\right)<+\infty
$$

We are now in position to apply Lemma A.2 in the appendix, with the sequences $\left(t_{k} \beta_{k-1}\right)$ and $\left(\frac{1}{\beta_{k-1}} W_{k}\right)$, respectively in place of $\left(\tau_{k}\right)$ and $\left(\varepsilon_{k}\right)$. We obtain that

$$
\frac{1}{\beta_{k-1}} W_{k}=o\left(\frac{1}{\sum_{i=1}^{k} t_{i} \beta_{i-1}}\right) \quad \text { as } k \rightarrow+\infty \text {. }
$$

Hence

$$
W_{k}=o\left(\frac{\beta_{k-1}}{\sum_{i=1}^{k} t_{i} \beta_{i-1}}\right) \quad \text { as } k \rightarrow+\infty
$$

which gives the claim.

As a direct consequence of Theorem 4.1, in the case without inertia, i.e. $\alpha_{k} \equiv 0$ (which gives $t_{k} \equiv 1$ ) we obtain the following result.

Corollary 4.2. Suppose that the sequence $\left(\beta_{k}\right)$ is non-decreasing. Then for any sequence $\left(x_{k}\right)$ generated by the proximal algorithm $x_{k+1}=\operatorname{prox}_{\beta_{k} \Phi}\left(x_{k}\right)$ we have the following estimates:

$$
\Phi\left(x_{k}\right)-\min _{\mathcal{H}} \Phi=o\left(\frac{1}{\sum_{i=1}^{k} \beta_{i-1}}\right) \text { and }\left\|x_{k}-x_{k-1}\right\|^{2}=o\left(\frac{\beta_{k-1}}{\sum_{i=1}^{k} \beta_{i-1}}\right) .
$$

When $\left(\beta_{k}\right)$ is non-decreasing, this notably improves the results obtained by Güler in [20, Theorem 3.1].

\section{Convergence of the iterates}

Let us now fix $x^{*} \in \operatorname{argmin} \Phi$, and define $h_{k}:=\frac{1}{2}\left\|x_{k}-x^{*}\right\|^{2}$. The sequence $\left(h_{k}\right)$ takes account of the anchoring of the sequence of iterates $\left(x_{k}\right)$ to the solution set. The next result will be useful for establishing the convergence of the iterates of the algorithm (IP) $)_{\alpha_{k}, \beta_{k}}$.

Proposition 5.1. For any $k \geq 1$, the following inequality holds

$$
h_{k+1}-h_{k}-\alpha_{k}\left(h_{k}-h_{k-1}\right) \leq \frac{1}{2}\left(\alpha_{k}^{2}+\alpha_{k}\right)\left\|x_{k}-x_{k-1}\right\|^{2}-\beta_{k}\left(\Phi\left(x_{k+1}\right)-\min _{\mathcal{H}} \Phi\right) .
$$

Proof. Observe that

$$
\begin{aligned}
\left\|y_{k}-x^{*}\right\|^{2} & =\left\|x_{k}+\alpha_{k}\left(x_{k}-x_{k-1}\right)-x^{*}\right\|^{2} \\
& =\left\|x_{k}-x^{*}\right\|^{2}+\alpha_{k}^{2}\left\|x_{k}-x_{k-1}\right\|^{2}+2 \alpha_{k}\left\langle x_{k}-x^{*}, x_{k}-x_{k-1}\right\rangle \\
& =\left\|x_{k}-x^{*}\right\|^{2}+\alpha_{k}^{2}\left\|x_{k}-x_{k-1}\right\|^{2}+\alpha_{k}\left\|x_{k}-x^{*}\right\|^{2}+\alpha_{k}\left\|x_{k}-x_{k-1}\right\|^{2}-\alpha_{k}\left\|x_{k-1}-x^{*}\right\|^{2} \\
& =\left\|x_{k}-x^{*}\right\|^{2}+\alpha_{k}\left(\left\|x_{k}-x^{*}\right\|^{2}-\left\|x_{k-1}-x^{*}\right\|^{2}\right)+\left(\alpha_{k}^{2}+\alpha_{k}\right)\left\|x_{k}-x_{k-1}\right\|^{2} \\
& =2\left[h_{k}+\alpha_{k}\left(h_{k}-h_{k-1}\right)\right]+\left(\alpha_{k}^{2}+\alpha_{k}\right)\left\|x_{k}-x_{k-1}\right\|^{2} .
\end{aligned}
$$


Set briefly $A_{k}=h_{k+1}-h_{k}-\alpha_{k}\left(h_{k}-h_{k-1}\right)=\frac{1}{2}\left\|x_{k+1}-x^{*}\right\|^{2}-\left[h_{k}+\alpha_{k}\left(h_{k}-h_{k-1}\right)\right]$. According to the above inequality, we get

$$
\begin{aligned}
A_{k} & =\frac{1}{2}\left\|x_{k+1}-x^{*}\right\|^{2}-\frac{1}{2}\left\|y_{k}-x^{*}\right\|^{2}+\frac{1}{2}\left(\alpha_{k}^{2}+\alpha_{k}\right)\left\|x_{k}-x_{k-1}\right\|^{2} \\
& =\left\langle x_{k+1}-y_{k}, \frac{1}{2}\left(x_{k+1}+y_{k}\right)-x^{*}\right\rangle+\frac{1}{2}\left(\alpha_{k}^{2}+\alpha_{k}\right)\left\|x_{k}-x_{k-1}\right\|^{2} \\
& =\left\langle x_{k+1}-y_{k}, y_{k}-x^{*}\right\rangle+\frac{1}{2}\left\|x_{k+1}-y_{k}\right\|^{2}+\frac{1}{2}\left(\alpha_{k}^{2}+\alpha_{k}\right)\left\|x_{k}-x_{k-1}\right\|^{2} .
\end{aligned}
$$

So, we obtain the equality

(37) $h_{k+1}-h_{k}-\alpha_{k}\left(h_{k}-h_{k-1}\right)=\frac{1}{2}\left(\alpha_{k}^{2}+\alpha_{k}\right)\left\|x_{k}-x_{k-1}\right\|^{2}+\left\langle y_{k}-x_{k+1}, x^{*}-y_{k}\right\rangle+\frac{1}{2}\left\|y_{k}-x_{k+1}\right\|^{2}$.

Now use that $x^{*} \in \operatorname{argmin} \Phi$. By definition of the proximal operator, $x_{k+1}=\operatorname{prox}_{\beta_{k} \Phi}\left(y_{k}\right)$ equivalently gives $\frac{1}{\beta_{k}}\left(y_{k}-x_{k+1}\right) \in \partial \Phi\left(x_{k+1}\right)$. The convex subdifferential inequality applied at $x_{k+1}$ gives

$$
\Phi\left(x^{*}\right) \geq \Phi\left(x_{k+1}\right)+\left\langle\frac{1}{\beta_{k}}\left(y_{k}-x_{k+1}\right), x^{*}-x_{k+1}\right\rangle .
$$

Since $\Phi\left(x^{*}\right)=\min _{\mathcal{H}} \Phi$, we infer that

$$
\left\langle y_{k}-x_{k+1}, x^{*}-x_{k+1}\right\rangle \leq-\beta_{k}\left(\Phi\left(x_{k+1}\right)-\min _{\mathcal{H}} \Phi\right) .
$$

Hence

$$
\left\|y_{k}-x_{k+1}\right\|^{2}+\left\langle y_{k}-x_{k+1}, x^{*}-y_{k}\right\rangle \leq-\beta_{k}\left(\Phi\left(x_{k+1}\right)-\min _{\mathcal{H}} \Phi\right) .
$$

Combining this inequality with (37) gives

$$
h_{k+1}-h_{k}-\alpha_{k}\left(h_{k}-h_{k-1}\right) \leq \frac{1}{2}\left(\alpha_{k}^{2}+\alpha_{k}\right)\left\|x_{k}-x_{k-1}\right\|^{2}-\beta_{k}\left(\Phi\left(x_{k+1}\right)-\min _{\mathcal{H}} \Phi\right),
$$

which completes the proof of Proposition 5.1.

We will also need the following estimate for the velocities which completes Theorem 3.2.

Proposition 5.2. Suppose that the sequences $\left(\alpha_{k}\right)$ and $\left(\beta_{k}\right)$ satisfy the condition $\left(K_{1, \alpha_{k}, \beta_{k}}^{+}\right)$and that the sequence $\left(\beta_{k}\right)$ is non-decreasing. Then, we have

$$
\sum_{k=1}^{+\infty} t_{k+1}\left\|x_{k}-x_{k-1}\right\|^{2}<+\infty
$$

Proof. Condition $\left(K_{1, \alpha_{k}, \beta_{k}}^{+}\right)$can be equivalently written as

$$
\left(t_{k+1}^{2}-t_{k}^{2}\right) \beta_{k}+t_{k}^{2}\left(\beta_{k}-\beta_{k-1}\right) \leq t_{k+1} \beta_{k}
$$

Since the sequence $\left(\beta_{k}\right)$ is non-decreasing, we infer $t_{k+1}^{2}-t_{k}^{2} \leq t_{k+1}$. Hence

$$
t_{k+1}-t_{k} \leq \frac{t_{k+1}}{t_{k+1}+t_{k}} \leq 1
$$

Since $1 \leq t_{k}$, we immediately obtain $t_{k+1} \leq 2 t_{k}$. So

$$
\sum_{k=1}^{+\infty} t_{k+1}\left\|x_{k}-x_{k-1}\right\|^{2} \leq 2 \sum_{k=1}^{+\infty} t_{k}\left\|x_{k}-x_{k-1}\right\|^{2}<+\infty,
$$

where the last inequality comes from Theorem 3.2.

Theorem 5.3. Assume that

$i)$ the sequences $\left(\alpha_{k}\right)$ and $\left(\beta_{k}\right)$ satisfy the condition $\left(K_{1, \alpha_{k}, \beta_{k}}^{+}\right)$;

ii) $\alpha_{k} \in[0,1]$ for every $k \geq 1$;

iii) the sequence $\left(\beta_{k}\right)$ is non-decreasing;

iv) $\sum_{k=1}^{\infty} t_{k} \beta_{k-1}=+\infty$.

Then, any sequence $\left(x_{k}\right)$ generated by the algorithm $(\mathrm{IP})_{\alpha_{k}, \beta_{k}}$ converges weakly, and its limit belongs to $\operatorname{argmin} \Phi$.

Proof. We apply the Opial lemma, see Lemma A.1. Assume that there exist $\bar{x} \in H$ and a sequence $\left(k_{n}\right)$ such that $k_{n} \rightarrow+\infty$, and $x_{k_{n}} \rightarrow \bar{x}$ weakly as $n \rightarrow+\infty$. Since the convex function $\Phi$ is lower semicontinuous, it is lower semicontinuous for the weak topology, hence satisfies

$$
\Phi(\bar{x}) \leq \liminf _{n \rightarrow+\infty} \Phi\left(x_{k_{n}}\right)=\lim _{k \rightarrow+\infty} \Phi\left(x_{k}\right)=\min _{\mathcal{H}} \Phi,
$$


where the last equality comes from Theorem 4.1. It ensues that $\bar{x} \in \operatorname{argmin} \Phi$, which shows the first point. Let us now fix $x^{*} \in \operatorname{argmin} \Phi$, and show that $\lim _{k \rightarrow+\infty}\left\|x_{k}-x^{*}\right\|$ exists. Set $h_{k}=\frac{1}{2}\left\|x_{k}-x^{*}\right\|^{2}$. From Proposition $5.1,\left(h_{k}\right)$ satisfies the following inequalities

$$
h_{k+1}-h_{k}-\alpha_{k}\left(h_{k}-h_{k-1}\right) \leq \frac{1}{2}\left(\alpha_{k}^{2}+\alpha_{k}\right)\left\|x_{k}-x_{k-1}\right\|^{2} \leq\left\|x_{k}-x_{k-1}\right\|^{2} \quad \text { since } \alpha_{k} \in[0,1] .
$$

Taking the positive part, we find

$$
\left(h_{k+1}-h_{k}\right)_{+} \leq \alpha_{k}\left(h_{k}-h_{k-1}\right)_{+}+\left\|x_{k}-x_{k-1}\right\|^{2} .
$$

By Proposition 5.2 we have $\sum_{k=1}^{+\infty} t_{k+1}\left\|x_{k}-x_{k-1}\right\|^{2}<+\infty$. By applying Lemma A.3 (appendix) with $a_{k}=$ $\left(h_{k}-h_{k-1}\right)_{+}$and $\omega_{k}=\left\|x_{k}-x_{k-1}\right\|^{2}$, we obtain $\sum_{k=1}^{+\infty}\left(h_{k}-h_{k-1}\right)_{+}<+\infty$. Since $\left(h_{k}\right)$ is nonnegative, this implies that $\lim _{k \rightarrow+\infty} h_{k}$ exists. The second point of the Opial lemma is shown, which ends the proof.

\section{LiNK With GÜLER'S ACCELERATED PROXIMAL ALGORITHM}

Let's recall Güler's accelerated proximal algorithm, see [21]. We modify the notations to adapt them to our framework. The proximal parameter noted $\lambda_{k}$ in Güler's article is noted here $\beta_{k}$, and the inertial parameter is denoted by $g_{k}$ in place of $\gamma_{k}$.

a) Initialization of $\nu_{0}$ and $A_{0}$.

b) Step $k$ :

- Choose $\beta_{k}>0$, and calculate $g_{k}>0$ by solving

- Define

$$
g_{k}^{2}+g_{k} A_{k} \beta_{k}-A_{k} \beta_{k}=0
$$

i) $y_{k}=\left(1-g_{k}\right) x_{k}+g_{k} \nu_{k}$;

ii) $x_{k+1}=\operatorname{prox}_{\beta_{k} \Phi}\left(y_{k}\right)$;

iii) $\nu_{k+1}=\nu_{k}+\frac{1}{g_{k}}\left(x_{k+1}-y_{k}\right)$;

iv) $A_{k+1}=\left(1-g_{k}\right) A_{k}$.

6.1. Güler's accelerated proximal algorithm as (IP $)_{\alpha_{k}, \beta_{k}}$. Let us show that Güler's proximal algorithm can be written as an inertial proximal algorithm (IP) $)_{\alpha_{k}, \beta_{k}}$. First verify that, for all $k \geq 1$

$$
\nu_{k}=x_{k-1}+\frac{1}{g_{k-1}}\left(x_{k}-x_{k-1}\right) \text {. }
$$

For this, we use an induction argument. Suppose (39) is satisfied at step $k$. Using successively (38) iii), (39), (38) $i$ ), and (39) again, we obtain

$$
\begin{aligned}
\nu_{k+1} & =\nu_{k}+\frac{1}{g_{k}}\left(x_{k+1}-y_{k}\right)=x_{k-1}+\frac{1}{g_{k-1}}\left(x_{k}-x_{k-1}\right)+\frac{1}{g_{k}}\left(x_{k+1}-y_{k}\right) \\
& =\frac{1}{g_{k}} x_{k+1}+x_{k-1}+\frac{1}{g_{k-1}}\left(x_{k}-x_{k-1}\right)-\frac{1}{g_{k}}\left(\left(1-g_{k}\right) x_{k}+g_{k} \nu_{k}\right) \\
& =\frac{1}{g_{k}} x_{k+1}-\frac{1-g_{k}}{g_{k}} x_{k}=x_{k}+\frac{1}{g_{k}}\left(x_{k+1}-x_{k}\right),
\end{aligned}
$$

which shows that (39) is satisfied at step $k+1$. Then, combining (38) $i$ ) with (39), we obtain

$$
\begin{aligned}
y_{k} & =\left(1-g_{k}\right) x_{k}+g_{k} \nu_{k}=\left(1-g_{k}\right) x_{k}+g_{k}\left(x_{k-1}+\frac{1}{g_{k-1}}\left(x_{k}-x_{k-1}\right)\right) \\
& =x_{k}+\left(\frac{g_{k}}{g_{k-1}}-g_{k}\right)\left(x_{k}-x_{k-1}\right) .
\end{aligned}
$$

Hence, Güler's proximal algorithm can be written as

$$
\left\{\begin{array}{l}
y_{k}=x_{k}+\alpha_{k}\left(x_{k}-x_{k-1}\right) \\
x_{k+1}=\operatorname{prox}_{\beta_{k} \Phi}\left(y_{k}\right),
\end{array}\right.
$$

with

$$
\alpha_{k}=g_{k}\left(\frac{1}{g_{k-1}}-1\right) .
$$

By construction of $g_{k}$, we have $g_{k}=\frac{1}{2}\left(-A_{k} \beta_{k}+\sqrt{\left(A_{k} \beta_{k}\right)^{2}+4 A_{k} \beta_{k}}\right)$, which gives $0 \leq g_{k}<1$. According to (41), we deduce that $\alpha_{k}>0$. As a result, the first formula of (40) defines an extrapolation operation. This 
makes Güler's algorithm an inertial proximal algorithm, within the framework of the algorithm (IP) ${\alpha_{k}, \beta_{k}}_{\text {. From }}$ item $i v$ ) in Güler's algorithm, we immediately get:

$$
A_{k}=A_{0} \prod_{j=0}^{k-1}\left(1-g_{j}\right) .
$$

From the second-order algebraic equation that defines $g_{k}$ and (38) $i v$ ), we have

$$
g_{k}^{2}=A_{k} \beta_{k}\left(1-g_{k}\right)=\beta_{k} A_{k+1} \text {. }
$$

Combining (42) with (43), we obtain the following relation:

$$
\beta_{k}=\frac{g_{k}^{2}}{A_{0} \prod_{j=0}^{k}\left(1-g_{j}\right)} .
$$

According to (41)-(42)-(44), we have obtained that all the parameters entering into Güler's algorithm can be expressed according to the single parameter $g_{k}$.

6.2. Convergence rate of Güler's accelerated proximal algorithm. Let us show that the convergence rate of Güler's accelerated proximal algorithm falls within the framework of Theorem 2.3. For this, we must make the link between the parameters used in Güler's algorithm and the parameter $t_{k}$ used in Theorem 2.3. We start from

$$
\alpha_{k}=g_{k}\left(\frac{1}{g_{k-1}}-1\right) .
$$

Let us show how to invert this relation. Set $\gamma_{k}=1-\alpha_{k}$. According to (45)

$$
\gamma_{k}=1-g_{k}\left(\frac{1}{g_{k-1}}-1\right)=g_{k}\left(1+\frac{1}{g_{k}}-\frac{1}{g_{k-1}}\right) .
$$

Set $w_{k}:=\frac{1}{g_{k}}$. The sequence $\left(w_{k}\right)$ safisfies the finite-difference equation

$$
1+w_{k}-w_{k-1}=\gamma_{k} w_{k}
$$

which gives, for all $k \geq 1$,

$$
w_{k-1}=1+\alpha_{k} w_{k} .
$$

Comparing with the relation (13) that defines the sequence $\left.t_{k}\right)$ in Lemma 2.1, we obtain $w_{k}=t_{k+1}$. Hence, for all $k \geq 1$

$$
g_{k}=\frac{1}{t_{k+1}} .
$$

We have now all the ingredients to verify that the condition

$$
\left(K_{1, \alpha_{k}, \beta_{k}}\right) \quad t_{k+1}^{2} \beta_{k}-t_{k}^{2} \beta_{k-1}-t_{k+1} \beta_{k} \leq 0 \quad \text { for every } k \geq 1 \text {, }
$$

is satisfied by Güler's algorithm. We have

$$
\begin{aligned}
t_{k+1}^{2} \beta_{k}-t_{k}^{2} \beta_{k-1}-t_{k+1} \beta_{k} & =t_{k+1} \beta_{k}\left(t_{k+1}-1\right)-t_{k}^{2} \beta_{k-1} \\
& =\frac{\beta_{k}}{g_{k}}\left(\frac{1-g_{k}}{g_{k}}\right)-\frac{\beta_{k-1}}{g_{k-1}^{2}} \\
& =\frac{g_{k}^{2}}{A_{0} \prod_{j=0}^{k}\left(1-g_{j}\right)} \frac{1}{g_{k}}\left(\frac{1-g_{k}}{g_{k}}\right)-\frac{1}{g_{k-1}^{2}} \frac{g_{k-1}^{2}}{A_{0} \prod_{j=0}^{k-1}\left(1-g_{j}\right)} \\
& =\frac{1}{A_{0} \prod_{j=0}^{k-1}\left(1-g_{j}\right)}-\frac{1}{A_{0} \prod_{j=0}^{k-1}\left(1-g_{j}\right)}=0 .
\end{aligned}
$$

So, Güler's accelerated proximal algorithm corresponds to taking equality in the condition $\left(K_{1, \alpha_{k}, \beta_{k}}\right)$. Let us summarize the above results.

Proposition 6.1. Güler's accelerated proximal algorithm is within the framework of algorithm (IP) ${\alpha_{k}, \beta_{k}}_{k}$ with $\alpha_{k}=g_{k}\left(\frac{1}{g_{k-1}}-1\right)$. It satisfies the condition $\left(K_{1, \alpha_{k}, \beta_{k}}\right)$ with equality instead of inequality, i.e., for all $k \geq 1$

$$
t_{k+1}^{2} \beta_{k}-t_{k}^{2} \beta_{k-1}-t_{k+1} \beta_{k}=0 .
$$

The following rate of convergence is satisfied: For every $k \geq 1$,

$$
\Phi\left(x_{k}\right)-\min _{\mathcal{H}} \Phi \leq \frac{C}{t_{k}^{2} \beta_{k-1}},
$$


with $C=t_{1}^{2} \beta_{0}\left(\Phi\left(x_{1}\right)-\min _{\mathcal{H}} \Phi\right)+\frac{1}{2}\left(d\left(x_{0}, \operatorname{argmin}_{\mathcal{H}} \Phi\right)^{2}+t_{1}^{2}\left\|x_{1}-x_{0}\right\|^{2}\right)$.

Moreover, for every $k \geq 1$,

$$
\Phi\left(x_{k}\right)-\min _{\mathcal{H}} \Phi=\mathcal{O}\left(\frac{1}{\sum_{j=1}^{k} t_{j} \beta_{j-1}}\right) .
$$

The above formulas can be expressed equivalently in terms of Güler's parameters thanks to the relation $t_{k}=\frac{1}{g_{k-1}}$ :

$$
\Phi\left(x_{k}\right)-\min _{\mathcal{H}} \Phi=\mathcal{O}\left(\frac{g_{k-1}^{2}}{\beta_{k-1}}\right) \quad \Phi\left(x_{k}\right)-\min _{\mathcal{H}} \Phi=\mathcal{O}\left(\frac{1}{\sum_{j=1}^{k} \frac{\beta_{j-1}}{g_{j-1}}}\right)
$$

Proof. The convergence rate given in (47) results from Theorem 2.3. Let us now exploit the fact that in the case of Güler's algorithm, the condition $\left(K_{1, \alpha_{k}, \beta_{k}}\right)$ is satisfied, with equality. By adding the equalities

$$
t_{j}^{2} \beta_{j-1}-t_{j-1}^{2} \beta_{j-2}=t_{j} \beta_{j-1}
$$

from $j=1$ to $k$, we get

$$
t_{k}^{2} \beta_{k-1}=\sum_{j=1}^{k} t_{j} \beta_{j-1}+t_{1} \beta_{0} .
$$

This gives (48), by just assuming that theses quantities are infinite. This is a minimal assumption to insure convergence of the algorithm.

Let's now compare the convergence rate given as above, which results from Theorem 2.3, with the convergence rate obtained by Güler in [21]

$$
\Phi\left(x_{k}\right)-\min _{\mathcal{H}} \Phi=\mathcal{O}\left(\frac{1}{\left(\sum_{j=1}^{k-1} \sqrt{\beta_{j}}\right)^{2}}\right) .
$$

The two above formulas give the same rate of convergence in most practical situations. Let's give a simple result where we can deduce the convergence rate obtained by Güler from the Proposition 6.1. Suppose that $\left(\beta_{k}\right)$ is a non-decreasing sequence, and that $t_{k} \geq C k$ for some positive constant $C$, and $k$ large enough. This last assumption is satisfied for the Nesterov type acceleration formula with $\alpha_{k}=1-\frac{\alpha}{k}$ and $\alpha \geq 3$, see [5]. Since $\left(\beta_{k}\right)$ is non-decreasing

$$
\left(\sum_{j=1}^{k-1} \sqrt{\beta_{j}}\right)^{2} \leq(k-1)^{2} \beta_{k-1}
$$

Since $t_{k} \geq C k$ for $k$ large enough, we deduce that (for some other constant $C$ )

$$
\frac{1}{\left(\sum_{j=1}^{k-1} \sqrt{\beta_{j}}\right)^{2}} \geq \frac{C}{t_{k}^{2} \beta_{k-1}}
$$

which gives the claim.

Remark 6.2. Güler's accelerated proximal algorithm corresponds to taking equality in $\left(K_{1, \alpha_{k}, \beta_{k}}\right)$. As such, it fits into the critical case, introduced historically by Nesterov, and for which the convergence of the iterates is still an open question. By contrast, as shown in Theorem 5.3, taking strict inequality (precisely assuming the condition $\left.\left(K_{1, \alpha_{k}, \beta_{k}}^{+}\right)\right)$gives the convergence of iterates, and makes it possible to pass from the capital $\mathcal{O}$ to small $o$ convergence rates. See [18] and [11] who initiated this type of result.

6.3. Dynamic interpretation of Güler's algorithm. Let's come with the dynamic interpretation of Güler's algorithm, as formulated in (40-41). According to the formulation (41) of $\alpha_{k}$ we get

$$
x_{k+1}+\beta_{k} \partial \Phi\left(x_{k+1}\right) \ni y_{k}=x_{k}+g_{k}\left(\frac{1}{g_{k-1}}-1\right)\left(x_{k}-x_{k-1}\right) .
$$

Equivalently,

$$
x_{k+1}-2 x_{k}+x_{k-1}+\left(g_{k}-\frac{g_{k}-g_{k-1}}{g_{k-1}}\right)\left(x_{k}-x_{k-1}\right)+\beta_{k} \partial \Phi\left(x_{k+1}\right)=0 .
$$

This can be interpreted as a time discretization of the second-order evolution equation (when $\Phi$ is smooth)

$$
\ddot{x}(t)+\left(g(t)-\frac{\dot{g}(t)}{g(t)}\right) \dot{x}(t)+\beta(t) \nabla \Phi(x(t))=0 .
$$


So, Güler's proximal algorithm is based on the damped inertial dynamic (IGS) ${ }_{\gamma, \beta}$ where the damping coefficient is expressed as

$$
\gamma(t)=g(t)-\frac{\dot{g}(t)}{g(t)}=g(t)\left(1+\frac{d}{d t}\left(\frac{1}{g}\right)(t)\right) .
$$

This makes the link with the next section, where we show that, in this context, the corresponding dynamic and proximal algorithms can be studied more directly: the conditions ensuring the rapid convergence of the algorithm can be expressed directly on the parameters $g_{k}$ and $\beta_{k}$. This avoids the use of the coefficients $t_{k}$ whose study requires additional analysis, see [5] for more details.

\section{A Class OF INERTial PROXimal ALGORITHMS}

Motivated by the results above, we consider the proximal algorithms that can be obtained (when $\Phi$ is smooth) by various temporal discretizations of the second-order evolution equation

$$
\ddot{x}(t)+g(t)\left(1+\frac{d}{d t}\left(\frac{1}{g}\right)(t)\right) \dot{x}(t)+\lambda(t) \nabla \Phi(x(t))=0 .
$$

We will see that, without loss of generality, the convergence analysis for the Inertial Proximal algorithm (IP) ${ }_{\alpha_{k}, \beta_{k}}$ can be developed within this setting. In doing so, we will introduce a class of inertial proximal algorithms, including the one studied in the previous sections, and which share similar convergence properties. Moreover, the convergence results can now be expressed directly on the parameters describing the algorithms.

\subsection{Link between the two descriptions.}

7.1.1. Continuous case. Let's show that taking $g(t)\left(1+\frac{d}{d t}\left(\frac{1}{g}\right)(t)\right)$ as the damping coefficient $\gamma(t)$ is not a restrictive assumption. To see this, given a continuous function $\gamma(\cdot)$, we have to integrate the differential equation

$$
g(t)\left(1+\frac{d}{d t}\left(\frac{1}{g}\right)(t)\right)=\gamma(t) .
$$

Take as a new unknown function $w:=\frac{1}{g}$. Then (53) is equivalent to solve the non-autonomous linear differential equation

$$
\dot{w}(t)-\gamma(t) w(t)=-1 .
$$

Set $p(t):=\exp \left(\int_{t_{0}}^{t} \gamma(\tau) d \tau\right)$ for $t \geq t_{0}$, then, following [4] and assuming that $\int_{t_{0}}^{+\infty} \frac{d s}{p(s)}<+\infty$, we obtain as a solution

$$
w(t)=p(t) \int_{t}^{\infty} \frac{1}{p(s)} d s .
$$

This is the function $\Gamma(\cdot)$ introduced in [4]. Hence $g(t)=\frac{1}{\Gamma(t)}$ is solution of (53). The general solution is $g(t)=\frac{1}{C p(t)+\Gamma(t)}$ with $\frac{1}{C}=\lim _{t \rightarrow+\infty} p(t) g(t)$. Let's summarize the above results in the following lemma.

Lemma 7.1. Let $\gamma:\left[t_{0},+\infty\left[\rightarrow \mathbb{R}\right.\right.$ be a positive continuous function. Set $p(t)=\exp \left(\int_{t_{0}}^{t} \gamma(\tau) d \tau\right)$ and assume $\int_{t_{0}}^{+\infty} \frac{d s}{p(s)}<+\infty$. Consider the following differential equation, where the unknown function is $g(\cdot)$ :

$$
g(t)\left(1+\frac{d}{d t}\left(\frac{1}{g}\right)(t)\right)=\gamma(t) .
$$

Then, the following non-negative continuously differentiable function $g(\cdot)$ is solution of $(54)$ :

$$
g(t)=\frac{1}{\Gamma(t)} \quad \text { where } \quad \Gamma(t)=p(t) \int_{t}^{\infty} \frac{1}{p(s)} d s .
$$

Precisely, it is the solution of (54) that satisfies $\lim _{t \rightarrow+\infty} \frac{1}{p(t) g(t)}=0$.

Note that the above definition of $\Gamma$ does not depend on the initial time $t_{0}$. When $\beta(t) \equiv \beta>0$ is fixed, fast convergence of the values is obtained in [4] under the condition $\gamma(t) \Gamma(t) \leq \frac{3}{2}$. According to (54) and (55), in terms of $g(t)$, this condition takes the equivalent form

$$
g(t)\left(1+\frac{d}{d t}\left(\frac{1}{g}\right)(t)\right) \frac{1}{g(t)}=1+\frac{d}{d t}\left(\frac{1}{g}\right)(t) \leq \frac{3}{2} .
$$

So, the condition $\gamma(t) \Gamma(t) \leq \frac{3}{2}$ becomes

$$
\frac{d}{d t}\left(\frac{1}{g}\right)(t) \leq \frac{1}{2}
$$


with the corresponding convergence rate of the values

$$
\Phi(x(t))-\min _{\mathcal{H}} \Phi=\mathcal{O}\left(g(t)^{2}\right) .
$$

This shows the obvious interest in formulating the damping coefficient in the form $g(t)\left(1+\frac{d}{d t}\left(\frac{1}{g}\right)(t)\right)$. There is no loss of generality, and the conditions for obtaining rapid convergence results can be formulated directly on the data $g$. For example, let's start with $g(t)=\frac{\alpha-1}{t}$. From (54), we immediately obtain $\gamma(t)=\frac{\alpha}{t}$. Then, (56)-(57) shows that the well-known condition $\alpha \geq 3$ provides the $\mathcal{O}\left(\frac{1}{t^{2}}\right)$ convergence rate of values (see [4], [14], [37]).

7.1.2. Discrete case. Similar results occur in the discrete case. Recall that the implicit temporal discretization of (52) gives

$$
x_{k+1}-2 x_{k}+x_{k-1}+g_{k}\left(1+\frac{1}{g_{k}}-\frac{1}{g_{k-1}}\right)\left(x_{k}-x_{k-1}\right)+\lambda_{k} \partial \Phi\left(x_{k+1}\right) \ni 0 .
$$

Equivalently

$$
\left\{\begin{array}{l}
y_{k}=x_{k}+\alpha_{k}\left(x_{k}-x_{k-1}\right) \\
x_{k+1}=\operatorname{prox}_{\lambda_{k} \Phi}\left(y_{k}\right)
\end{array}\right.
$$

where

$$
\alpha_{k}=g_{k}\left(\frac{1}{g_{k-1}}-1\right) .
$$

Set $\gamma_{k}=1-\alpha_{k}$. This gives $\gamma_{k}=g_{k}\left(1+\frac{1}{g_{k}}-\frac{1}{g_{k-1}}\right)$. As shown in the previous section, inverting this relation gives, for all $k \geq 1, g_{k}=\frac{1}{t_{k+1}}$.

Let's summarize the above results in the following lemma.

Lemma 7.2. Let $\left(\gamma_{k}\right)$ be a sequence of non-negative numbers. Consider the following finite-difference equation, where the unknown sequence is $\left(g_{k}\right)$

$$
g_{k}\left(1+\frac{1}{g_{k}}-\frac{1}{g_{k-1}}\right)=\gamma_{k} .
$$

Then, the following sequence of non-negative numbers is solution of (61):

$$
g_{k}=\frac{1}{t_{k+1}} \quad \text { where } t_{k}=1+\sum_{i=k}^{+\infty} \prod_{j=k}^{i} \alpha_{j} .
$$

Following Theorem 1.1 recalled in the introduction, when $\beta_{k} \equiv \beta>0$ is fixed, we obtain a fast convergence of the values (see [5]) under the condition

$$
\left(K_{1}\right) \quad \forall k \geq 1, \quad t_{k+1}^{2}-t_{k}^{2} \leq t_{k+1} .
$$

According to the above lemma this condition can be equivalently formulated in terms of $g_{k}$ as

$$
g_{k} \geq 1-\left(\frac{g_{k}}{g_{k-1}}\right)^{2}
$$

with the corresponding convergence rate of the values

$$
\Phi\left(x_{k}\right)-\min _{\mathcal{H}} \Phi=\mathcal{O}\left(g_{k}^{2}\right) .
$$

Starting from $g_{k}=\frac{\alpha-1}{k},(61)$ immediately gives $\gamma_{k}=\frac{\alpha}{k}$. Then (63)-(64) shows that the well-known condition $\alpha \geq 3$ provides the $\mathcal{O}\left(\frac{1}{k^{2}}\right)$ convergence rate of values, a classical result (see [5], [8], [11], [18], [37]).

7.2. A parametrized family of proximal inertial algorithms. Let us start from the second-order evolution equation (52) and introduce various temporal discretizations. When considering the implicit discretization for the potential term, which gives proximal algorithms, we can take a general convex lower semicontinuous proper function $\Phi$. As novelty, we introduce a parameter $\theta \in[0,1]$ which takes into account different discretizations of the damping term: for $k \geq 1$,

$$
\begin{aligned}
\left(x_{k+1}-2 x_{k}+x_{k-1}\right) & +g_{k}(1-\theta)\left(1+\frac{1}{g_{k+1}}-\frac{1}{g_{k}}\right)\left(x_{k+1}-x_{k}\right) \\
& +g_{k} \theta\left(1+\frac{1}{g_{k}}-\frac{1}{g_{k-1}}\right)\left(x_{k}-x_{k-1}\right)+\lambda_{k} \partial \Phi\left(x_{k+1}\right) \ni 0 .
\end{aligned}
$$


For $\theta=1$, we recover the algorithm considered in the previous sections. After dividing by $g_{k}$, we obtain

$$
\begin{array}{ll}
\left(\frac{1}{g_{k}}+(1-\theta)\left(1+\frac{1}{g_{k+1}}-\frac{1}{g_{k}}\right)\right) & \left(x_{k+1}-x_{k}\right) \\
-\left(\frac{1}{g_{k}}+\theta\left(\frac{1}{g_{k-1}}-\frac{1}{g_{k}}-1\right)\right) & \left(x_{k}-x_{k-1}\right)+\frac{\lambda_{k}}{g_{k}} \partial \Phi\left(x_{k+1}\right) \ni 0 .
\end{array}
$$

Set, for $k \geq 1, \theta_{k}:=\frac{1}{g_{k}}+\theta\left(\frac{1}{g_{k-1}}-\frac{1}{g_{k}}-1\right)$. Note that

$$
\frac{1}{g_{k}}+(1-\theta)\left(1+\frac{1}{g_{k+1}}-\frac{1}{g_{k}}\right)=1+\theta_{k+1}
$$

So, we can reformulate the above algorithm in the condensed form

$$
x_{k+1}+\frac{\lambda_{k}}{g_{k}\left(1+\theta_{k+1}\right)} \partial \Phi\left(x_{k+1}\right) \ni x_{k}+\frac{\theta_{k}}{1+\theta_{k+1}}\left(x_{k}-x_{k-1}\right) .
$$

This gives the Inertial Proximal algorithm with parameter $\theta$ :

\begin{aligned} & \hline Inertial Proximal algorithm with parameter $\theta \\ &(\mathrm{IP})_{\alpha_{k}, \beta_{k}, \theta} \quad\left\{\begin{array}{l}y_{k}=x_{k}+\alpha_{k}\left(x_{k}-x_{k-1}\right) \\ x_{k+1}=\operatorname{prox}_{\beta_{k} \Phi}\left(y_{k}\right) \\ \alpha_{k}:=\frac{\theta_{k}}{1+\theta_{k+1}} ; \\ \beta_{k}:=\frac{\lambda_{k}}{g_{k}\left(1+\theta_{k+1}\right)} ; \\ \theta_{k}:=\frac{1}{g_{k}}+\theta\left(\frac{1}{g_{k-1}}-\frac{1}{g_{k}}-1\right)\end{array}\right. \\ &$\hline\end{aligned}

$(\mathrm{IP})_{\alpha_{k}, \beta_{k}, \theta}$ fits into the general framework of (IP $)_{\alpha_{k}, \beta_{k}}$, with extrapolation parameters and proximal parameters written in a specific form.

7.3. Convergence rates. An analysis parallel to that developed in the previous sections can be developed. We only detail the study of the rapid convergence of values.

Theorem 7.3. Consider the inertial proximal algorithm (IP $)_{\alpha_{k}, \beta_{k}, \theta}$ where $\alpha_{k}:=\frac{\theta_{k}}{1+\theta_{k+1}}, \beta_{k}:=\frac{\lambda_{k}}{g_{k}\left(1+\theta_{k+1}\right)}$, and $\theta_{k}:=\frac{1}{g_{k}}+\theta\left(\frac{1}{g_{k-1}}-\frac{1}{g_{k}}-1\right)$. Suppose that $0<g_{k} \leq 1,0 \leq \theta \leq 1$, and the parameters $\left(g_{k}\right)$, $\left(\lambda_{k}\right)$ and $\theta$ satisfy the growth condition: there exists $k_{1} \in \mathbb{N}$ such that for all $k \geq k_{1}$

$\left(K_{\left.1, g_{k}, \lambda_{k}, \theta\right)}\right.$

$$
\lambda_{k+1} \leq \frac{g_{k+1}}{g_{k}} \frac{\theta_{k+1}+1}{\theta_{k+2}} \lambda_{k} .
$$

Then, for any sequence $\left(x_{k}\right)$ generated by the algorithm $(\mathrm{IP})_{\alpha_{k}, \beta_{k}, \theta}$, we have

$$
\left\{\begin{array}{l}
\text { (i) } \Phi\left(x_{k}\right)-\min _{\mathcal{H}} \Phi=\mathcal{O}\left(\frac{g_{k-1}}{\lambda_{k-1}\left(1+\theta_{k}\right)}\right) \text {, as } k \rightarrow+\infty ; \\
\text { (ii) } \sum_{k \geq 1} \beta_{k, \theta}\left(\Phi\left(x_{k}\right)-\min _{\mathcal{H}} \Phi\right)<+\infty \\
\text { where } \beta_{k, \theta}:=\frac{\lambda_{k-1}}{g_{k-1}}\left(1+\theta_{k}\right)-\frac{\lambda_{k}}{g_{k}} \theta_{k+1} \text { is non-negative by }\left(K_{1, g_{k}, \lambda_{k}, \theta}\right) .
\end{array}\right.
$$

The proof is similar to that of the more general perturbed case (Theorem 8.1).

7.4. Some examples. Depending on the choice of the parameter $\theta$, we obtain a specific algorithm, with its convergence rate. The classical situation (considered below) corresponds to the explicit discretization $(\theta=1)$ of the damping term. Let's consider the following cases of particular interest:

a) Case $\theta=1$ : it corresponds to the explicit discretization of the damping term

$$
\left(x_{k+1}-2 x_{k}+x_{k-1}\right)+g_{k}\left(1+\frac{1}{g_{k}}-\frac{1}{g_{k-1}}\right)\left(x_{k}-x_{k-1}\right)+\beta_{k} \partial \Phi\left(x_{k+1}\right) \ni 0 .
$$


This is precisely the algorithm studied in the previous section with $\alpha_{k}=g_{k}\left(\frac{1}{g_{k-1}}-1\right)$. Let us compare the result obtained by applying respectively Theorem 2.3 (using the $t_{k}$ ) and Theorem 7.3 (using the $g_{k}$ in an explicit form). On the one hand, according to $g_{k}=\frac{1}{t_{k+1}}$, the growth condition $\left(K_{1, \alpha_{k}, \beta_{k}}\right)$ of Theorem 2.3 can be written

$$
\beta_{k+1} \leq \frac{g_{k+1}}{g_{k}^{2}\left(\frac{1}{g_{k+1}}-1\right)} \beta_{k} .
$$

Making $\theta=1$ in the formula giving the parameters, we have

$$
\theta_{k}:=\frac{1}{g_{k}}+\left(\frac{1}{g_{k-1}}-\frac{1}{g_{k}}-1\right)=\frac{1}{g_{k-1}}-1 .
$$

Hence $\theta_{k+1}+1=\frac{1}{g_{k}}$, which gives $\beta_{k}=\lambda_{k}$. The formula $\left(K_{1, g_{k}, \lambda_{k}, \theta}\right)$ in Theorem 7.3 becomes

$$
\beta_{k+1} \leq \frac{g_{k+1}}{g_{k}} \frac{\frac{1}{g_{k}}}{\frac{1}{g_{k+1}}-1} \beta_{k}=\frac{g_{k+1}}{g_{k}^{2}\left(\frac{1}{g_{k+1}}-1\right)} \beta_{k} .
$$

So we recover the same growth condition. Let us now compare the convergence rates. Theorem 2.3 gives

$$
\Phi\left(x_{k}\right)-\min _{\mathcal{H}} \Phi=\mathcal{O}\left(\frac{1}{t_{k}^{2} \beta_{k-1}}\right) .
$$

Theorem 7.3 gives

$$
\Phi\left(x_{k}\right)-\min _{\mathcal{H}} \Phi=\mathcal{O}\left(\frac{g_{k-1}}{\lambda_{k-1}\left(1+\theta_{k}\right)}\right) .
$$

From $\theta_{k}+1=\frac{1}{g_{k-1}}$ we get

$$
\frac{g_{k-1}}{\lambda_{k-1}\left(1+\theta_{k}\right)}=\frac{g_{k-1}^{2}}{\lambda_{k-1}}=\frac{1}{t_{k}^{2} \beta_{k-1}} .
$$

So, we recover the same convergence rate.

b) Case $\theta=0$ : it corresponds to the implicit discretization of the damping term

$$
\left(x_{k+1}-2 x_{k}+x_{k-1}\right)+g_{k}\left(1+\frac{1}{g_{k+1}}-\frac{1}{\alpha_{k}}\right)\left(x_{k+1}-x_{k}\right)+\beta_{k} \partial \Phi\left(x_{k+1}\right) \ni 0 .
$$

We have $\theta_{k}=\frac{1}{g_{k}}$, which gives algorithm (IP) ${\alpha_{k}, \beta_{k}, \theta}_{\theta}$ with $\alpha_{k}=\frac{g_{k+1}}{g_{k}\left(1+g_{k+1}\right)}$ and $\beta_{k}=\frac{\lambda_{k} g_{k+1}}{g_{k}\left(1+g_{k+1}\right)}$. Theorem 7.3 gives that, under the condition $\left(K_{1, g_{k}, \lambda_{k}, \theta}\right)$

$$
\lambda_{k+1} \leq \frac{\left(1+g_{k+1}\right) g_{k+2}}{g_{k}} \lambda_{k}
$$

we have

$$
\Phi\left(x_{k}\right)-\min _{\mathcal{H}} \Phi=\mathcal{O}\left(\frac{g_{k} g_{k-1}}{\lambda_{k-1}\left(1+g_{k}\right)}\right), \text { as } k \rightarrow+\infty ;
$$

Consider the particular case $g_{k}=\frac{\alpha-1}{k-1}$. This gives $\alpha_{k}=\frac{k-1}{k+\alpha-1}$, which corresponds to a variant of the Nesterov acceleration scheme considered in [8], [18], [37]. An elementary calculation shows that the growth condition above and the corresponding convergence rate give results comparable to those of the explicit case.

\section{Stability With RESPECT TO PERTURbations, ERrors}

Consider the perturbed version of the evolution equation (52)

$$
\ddot{x}(t)+g(t)\left(1+\frac{d}{d t}\left(\frac{1}{g}\right)(t)\right) \dot{x}(t)+\lambda(t) \nabla \Phi(x(t))=e(t)
$$

where the second member of (70), denoted by $e(\cdot)$, can be interpreted as an external action on the system, a perturbation, or a control term. By following a parallel approach to the time discretization procedure described in section 7.2 , we obtain : for $k \geq 1$,

$$
\begin{aligned}
\left(x_{k+1}-2 x_{k}+x_{k-1}\right) & +g_{k}(1-\theta)\left(1+\frac{1}{g_{k+1}}-\frac{1}{g_{k}}\right)\left(x_{k+1}-x_{k}\right) \\
& +g_{k} \theta\left(1+\frac{1}{g_{k}}-\frac{1}{g_{k-1}}\right)\left(x_{k}-x_{k-1}\right)+\lambda_{k} \partial \Phi\left(x_{k+1}\right) \ni e_{k}
\end{aligned}
$$


From the algorithmic point of view, the sequence $\left(e_{k}\right)$ of elements of $\mathcal{H}$ takes into account the presence of perturbations, approximations, or errors. After dividing by $g_{k}$, we obtain

$$
\begin{array}{ll}
\left(\frac{1}{g_{k}}+(1-\theta)\left(1+\frac{1}{g_{k+1}}-\frac{1}{g_{k}}\right)\right) & \left(x_{k+1}-x_{k}\right) \\
-\left(\frac{1}{g_{k}}+\theta\left(\frac{1}{g_{k-1}}-\frac{1}{g_{k}}-1\right)\right) & \left(x_{k}-x_{k-1}\right)+\frac{\lambda_{k}}{g_{k}} \partial \Phi\left(x_{k+1}\right) \ni \frac{1}{g_{k}} e_{k} .
\end{array}
$$

Set, for $k \geq 1$

$$
\theta_{k}:=\frac{1}{g_{k}}+\theta\left(\frac{1}{g_{k-1}}-\frac{1}{g_{k}}-1\right)
$$

Note that

$$
\frac{1}{g_{k}}+(1-\theta)\left(1+\frac{1}{g_{k+1}}-\frac{1}{g_{k}}\right)=1+\theta_{k+1} .
$$

So, we can reformulate the above algorithm in the condensed form

$$
\left(1+\theta_{k+1}\right)\left(x_{k+1}-x_{k}\right)+\frac{\lambda_{k}}{g_{k}} \partial \Phi\left(x_{k+1}\right) \ni \theta_{k}\left(x_{k}-x_{k-1}\right)+\frac{1}{g_{k}} e_{k}
$$

Equivalently,

$$
x_{k+1}+\frac{\lambda_{k}}{g_{k}\left(1+\theta_{k+1}\right)} \partial \Phi\left(x_{k+1}\right) \ni x_{k}+\frac{\theta_{k}}{1+\theta_{k+1}}\left(x_{k}-x_{k-1}\right)+\frac{1}{g_{k}\left(1+\theta_{k+1}\right)} e_{k} .
$$

This gives the Inertial Proximal algorithm with parameter $\theta$ and error $r_{k},(\mathrm{IP})_{\alpha_{k}, \beta_{k}, r_{k}, \theta}$ for short

$$
\begin{aligned}
& \text { Inertial Proximal Algorithm with parameter } \theta \text { and error } r_{k} \text {. } \\
& (\mathrm{IP})_{\alpha_{k}, \beta_{k}, r_{k}, \theta}\left\{\begin{array}{l}
y_{k}=x_{k}+\alpha_{k}\left(x_{k}-x_{k-1}\right) \\
x_{k+1}=\operatorname{prox}_{\beta_{k} \Phi}\left(y_{k}-r_{k}\right),
\end{array}\right. \\
& \alpha_{k}:=\frac{\theta_{k}}{1+\theta_{k+1}} \\
& \beta_{k}:=\frac{\lambda_{k}}{g_{k}\left(1+\theta_{k+1}\right)} \\
& r_{k}=-\frac{1}{g_{k}\left(1+\theta_{k+1}\right)} e_{k} \\
& \theta_{k}:=\frac{1}{g_{k}}+\theta\left(\frac{1}{g_{k-1}}-\frac{1}{g_{k}}-1\right) \text {. }
\end{aligned}
$$

The following result extends Theorem 2.3 to the perturbed case.

Theorem 8.1. Consider the inertial proximal algorithm (IP) ${ }_{\alpha_{k}, \beta_{k}, r_{k}, \theta}$. Suppose that $0<g_{k} \leq 1,0 \leq \theta \leq 1$, and the parameters $\left(g_{k}\right),\left(\lambda_{k}\right)$ and $\theta$ satisfy the growth condition: there exists $k_{1} \in \mathbb{N}$ such that for all $k \geq k_{1}$

$\left(K_{\left.1, g_{k}, \lambda_{k}, \theta\right)}\right.$

$$
\lambda_{k+1} \leq \frac{g_{k+1}}{g_{k}} \frac{\theta_{k+1}+1}{\theta_{k+2}} \lambda_{k} .
$$

Suppose that the sequence $\left(r_{k}\right)$ satisfies the summability property

$$
\sum_{k \geq 1}\left(1+\theta_{k+1}\right)\left\|r_{k}\right\|<+\infty
$$

Then, for any sequence $\left(x_{k}\right)$ generated by the algorithm (IP $)_{\alpha_{k}, \beta_{k}, r_{k}, \theta}$, we have

$$
\left\{\begin{array}{l}
\text { (i) } \Phi\left(x_{k}\right)-\min _{\mathcal{H}} \Phi=\mathcal{O}\left(\frac{g_{k-1}}{\lambda_{k-1}\left(1+\theta_{k}\right)}\right) \text {, as } k \rightarrow+\infty \\
\text { (ii) } \sum_{k \geq 1} \beta_{k, \theta}\left(\Phi\left(x_{k}\right)-\min _{\mathcal{H}} \Phi\right)<+\infty \\
\text { where } \beta_{k, \theta}:=\frac{\lambda_{k-1}}{g_{k-1}}\left(1+\theta_{k}\right)-\frac{\lambda_{k}}{g_{k}} \theta_{k+1} \text { is non-negative by }\left(K_{1, g_{k}, \lambda_{k}, \theta}\right) .
\end{array}\right.
$$

Proof. To make the presentation simpler, without loss of generality, we take $k_{1}=1$. By definition of the proximal operator, the iteration at step $k$ of the algorithm (IP) ${ }_{\alpha_{k}, \beta_{k}, \theta}$ is written

$$
\frac{1}{\beta_{k}}\left(y_{k}-x_{k+1}-r_{k}\right) \in \partial \Phi\left(x_{k+1}\right) .
$$


Equivalently, we have the following subdifferential inequalities: for any $x \in \mathcal{H}$

$$
\Phi(x) \geq \Phi\left(x_{k+1}\right)+\frac{1}{\beta_{k}}\left\langle x-x_{k+1}, y_{k}-x_{k+1}\right\rangle-\frac{1}{\beta_{k}}\left\langle x-x_{k+1}, r_{k}\right\rangle .
$$

Let us write successively inequality (75) at $x=x_{k}$ and $x=x^{*} \in \operatorname{argmin} \Phi$. We obtain the two inequalities

$$
\begin{aligned}
& \Phi\left(x_{k}\right) \geq \Phi\left(x_{k+1}\right)+\frac{1}{\beta_{k}}\left\langle x_{k}-x_{k+1}, y_{k}-x_{k+1}\right\rangle-\frac{1}{\beta_{k}}\left\langle x_{k}-x_{k+1}, r_{k}\right\rangle, \\
& \Phi\left(x^{*}\right) \geq \Phi\left(x_{k+1}\right)+\frac{1}{\beta_{k}}\left\langle x^{*}-x_{k+1}, y_{k}-x_{k+1}\right\rangle-\frac{1}{\beta_{k}}\left\langle x^{*}-x_{k+1}, r_{k}\right\rangle .
\end{aligned}
$$

Using $x_{k}-x_{k+1}=x_{k}-y_{k}+y_{k}-x_{k+1}$ in (76) and $x^{*}-x_{k+1}=x^{*}-y_{k}+y_{k}-x_{k+1}$ in (77) we obtain

$$
\begin{gathered}
\Phi\left(x_{k}\right) \geq \Phi\left(x_{k+1}\right)+\frac{1}{\beta_{k}}\left\langle x_{k}-y_{k}, y_{k}-x_{k+1}\right\rangle-\frac{1}{\beta_{k}}\left\langle x_{k}-x_{k+1}, r_{k}\right\rangle+\frac{1}{\beta_{k}}\left\|y_{k}-x_{k+1}\right\|^{2}, \\
\Phi\left(x^{*}\right) \geq \Phi\left(x_{k+1}\right)+\frac{1}{\beta_{k}}\left\langle x^{*}-y_{k}, y_{k}-x_{k+1}\right\rangle-\frac{1}{\beta_{k}}\left\langle x^{*}-x_{k+1}, r_{k}\right\rangle+\frac{1}{\beta_{k}}\left\|y_{k}-x_{k+1}\right\|^{2} .
\end{gathered}
$$

Multiplying (78) by $\frac{\theta_{k}}{\alpha_{k}}-1 \geq 0$, then adding (79), we derive that

$$
\begin{aligned}
\left(\frac{\theta_{k}}{\alpha_{k}}-1\right)\left(\Phi\left(x_{k}\right)\right. & \left.-\Phi\left(x^{*}\right)\right) \geq \frac{\theta_{k}}{\alpha_{k}}\left(\Phi\left(x_{k+1}\right)-\Phi\left(x^{*}\right)\right)+\frac{\theta_{k}}{\alpha_{k} \beta_{k}}\left\|y_{k}-x_{k+1}\right\|^{2} \\
& +\frac{1}{\beta_{k}}\left\langle x_{k+1}-y_{k},\left(\frac{\theta_{k}}{\alpha_{k}}-1\right)\left(y_{k}-x_{k}\right)+y_{k}-x^{*}\right\rangle \\
& +\frac{1}{\beta_{k}}\left\langle\left(\frac{\theta_{k}}{\alpha_{k}}-1\right)\left(x_{k+1}-x_{k}\right)+x_{k+1}-x^{*}, r_{k}\right\rangle .
\end{aligned}
$$

By definition of $y_{k}$ we have

$$
\begin{aligned}
\left(\frac{\theta_{k}}{\alpha_{k}}-1\right)\left(y_{k}-x_{k}\right)+y_{k} & =\left(\frac{\theta_{k}}{\alpha_{k}}-1\right) \alpha_{k}\left(x_{k}-x_{k-1}\right)+x_{k}+\alpha_{k}\left(x_{k}-x_{k-1}\right) \\
& =x_{k}+\theta_{k}\left(x_{k}-x_{k-1}\right)=z_{k}
\end{aligned}
$$

where $z_{k}:=x_{k}+\theta_{k}\left(x_{k}-x_{k-1}\right)$. Moreover

$$
\left(\frac{\theta_{k}}{\alpha_{k}}-1\right)\left(x_{k+1}-x_{k}\right)+x_{k+1}=\theta_{k+1}\left(x_{k+1}-x_{k}\right)+x_{k+1}=z_{k+1} .
$$

We then deduce from (80) that

$$
\begin{aligned}
\left(\frac{\theta_{k}}{\alpha_{k}}-1\right)\left(\Phi\left(x_{k}\right)-\Phi\left(x^{*}\right)\right) & \geq \frac{\theta_{k}}{\alpha_{k}}\left(\Phi\left(x_{k+1}\right)-\Phi\left(x^{*}\right)\right)+\frac{1}{\beta_{k}}\left\langle x_{k+1}-y_{k}, z_{k}-x^{*}\right\rangle \\
& +\frac{1}{\beta_{k}}\left\langle r_{k}, z_{k+1}-x^{*}\right\rangle+\frac{\theta_{k}}{\alpha_{k} \beta_{k}}\left\|y_{k}-x_{k+1}\right\|^{2} .
\end{aligned}
$$

Equivalently, after multiplication by $\beta_{k}$

$$
\begin{aligned}
& \beta_{k}\left(\frac{\theta_{k}}{\alpha_{k}}-1\right)\left(\Phi\left(x_{k}\right)-\Phi\left(x^{*}\right)\right) \geq \frac{\beta_{k} \theta_{k}}{\alpha_{k}}\left(\Phi\left(x_{k+1}\right)-\Phi\left(x^{*}\right)\right) \\
& +\left\langle x_{k+1}-y_{k}, z_{k}-x^{*}\right\rangle+\left\langle r_{k}, z_{k+1}-x^{*}\right\rangle+\frac{\theta_{k}}{\alpha_{k}}\left\|y_{k}-x_{k+1}\right\|^{2} .
\end{aligned}
$$

To write (81) in a recursive form, we use $z_{k+1}-z_{k}=\left(1+\theta_{k+1}\right)\left(x_{k+1}-y_{k}\right)$. It ensues that

$$
\left\|z_{k+1}-x^{*}\right\|^{2}=\left\|z_{k}-x^{*}\right\|^{2}+2\left(1+\theta_{k+1}\right)\left\langle x_{k+1}-y_{k}, z_{k}-x^{*}\right\rangle+\left(1+\theta_{k+1}\right)^{2}\left\|x_{k+1}-y_{k}\right\|^{2},
$$

which gives

$$
\left\langle x_{k+1}-y_{k}, z_{k}-x^{*}\right\rangle=\frac{1}{2\left(1+\theta_{k+1}\right)}\left(\left\|z_{k+1}-x^{*}\right\|^{2}-\left\|z_{k}-x^{*}\right\|^{2}\right)-\frac{\left(1+\theta_{k+1}\right)}{2}\left\|x_{k+1}-y_{k}\right\|^{2} .
$$

Using this equality in (81), we find

$$
\begin{aligned}
\beta_{k}\left(\frac{\theta_{k}}{\alpha_{k}}-1\right)\left(\Phi\left(x_{k}\right)-\Phi\left(x^{*}\right)\right) & \geq \frac{\beta_{k} \theta_{k}}{\alpha_{k}}\left(\Phi\left(x_{k+1}\right)-\Phi\left(x^{*}\right)\right)+\left\langle r_{k}, z_{k+1}-x^{*}\right\rangle \\
& +\frac{1}{2\left(1+\theta_{k+1}\right)}\left(\left\|z_{k+1}-x^{*}\right\|^{2}-\left\|z_{k}-x^{*}\right\|^{2}\right)+\frac{\left(1+\theta_{k+1}\right)}{2}\left\|x_{k+1}-y_{k}\right\|^{2},
\end{aligned}
$$


where we have used $\frac{\theta_{k}}{\alpha_{k}}-\frac{\left(1+\theta_{k+1}\right)}{2}=\frac{\left(1+\theta_{k+1}\right)}{2}$ (a consequence of the definition of $\alpha_{k}$ ). After multiplication by $\left(1+\theta_{k+1}\right)$, and neglecting the non-negative term $\frac{\left(1+\theta_{k+1}\right)}{2}\left\|x_{k+1}-y_{k}\right\|^{2}$, we obtain

$$
\begin{aligned}
& \beta_{k}\left(1+\theta_{k+1}\right)\left(\frac{\theta_{k}}{\alpha_{k}}-1\right)\left(\Phi\left(x_{k}\right)-\Phi\left(x^{*}\right)\right)+\frac{1}{2}\left\|z_{k}-x^{*}\right\|^{2} \\
& \geq \frac{\beta_{k}\left(1+\theta_{k+1}\right) \theta_{k}}{\alpha_{k}}\left(\Phi\left(x_{k+1}\right)-\Phi\left(x^{*}\right)\right)+\frac{1}{2}\left\|z_{k+1}-x^{*}\right\|^{2}+\left(1+\theta_{k+1}\right)\left\langle r_{k}, z_{k+1}-x^{*}\right\rangle .
\end{aligned}
$$

According to $\beta_{k}\left(1+\theta_{k+1}\right)=\frac{\lambda_{k}}{g_{k}}$, and $\frac{\theta_{k}}{\alpha_{k}}-1=\theta_{k+1}$ we have

$$
\beta_{k}\left(1+\theta_{k+1}\right)\left(\frac{\theta_{k}}{\alpha_{k}}-1\right)=\frac{\lambda_{k}}{g_{k}} \theta_{k+1} .
$$

Hence, (82) can be equivalently written as

$$
\begin{aligned}
\frac{\lambda_{k}}{g_{k}} \theta_{k+1}\left(\Phi\left(x_{k}\right)-\Phi\left(x^{*}\right)\right) & +\frac{1}{2}\left\|z_{k}-x^{*}\right\|^{2} \geq \frac{\lambda_{k}}{g_{k}}\left(1+\theta_{k+1}\right)\left(\Phi\left(x_{k+1}\right)-\Phi\left(x^{*}\right)\right) \\
& +\frac{1}{2}\left\|z_{k+1}-x^{*}\right\|^{2}+\left(1+\theta_{k+1}\right)\left\langle r_{k}, z_{k+1}-x^{*}\right\rangle .
\end{aligned}
$$

This naturally leads us to introduce the sequence $\left(\mathcal{E}_{k}\right)$

$$
\mathcal{E}_{k}=\frac{\lambda_{k-1}}{g_{k-1}}\left(1+\theta_{k}\right)\left(\Phi\left(x_{k}\right)-\Phi\left(x^{*}\right)\right)+\frac{1}{2}\left\|z_{k}-x^{*}\right\|^{2} .
$$

Thus, we have obtained the following inequality

$$
\mathcal{E}_{k} \geq \mathcal{E}_{k+1}+\left(\frac{\lambda_{k-1}}{g_{k-1}}\left(1+\theta_{k}\right)-\frac{\lambda_{k}}{g_{k}} \theta_{k+1}\right)\left(\Phi\left(x_{k}\right)-\min _{\mathcal{H}} \Phi\right)+\left(1+\theta_{k+1}\right)\left\langle r_{k}, z_{k+1}-x^{*}\right\rangle .
$$

Under condition $K_{1, g_{k}, \lambda_{k}, \theta}$ we have $\frac{\lambda_{k-1}}{g_{k-1}}\left(1+\theta_{k}\right)-\frac{\lambda_{k}}{g_{k}} \theta_{k+1} \geq 0$. Hence,

$$
\left(1+\theta_{k+1}\right)\left\|r_{k}\right\| \times\left\|z_{k+1}-x^{*}\right\|+\mathcal{E}_{k} \geq \mathcal{E}_{k+1} .
$$

By summing inequalities (85) from $j=1$ to $k-1$, we obtain

$$
\mathcal{E}_{k} \leq \mathcal{E}_{1}+\sum_{j=2}^{k}\left(1+\theta_{j}\right)\left\|r_{j-1}\right\| \times\left\|z_{j}-x^{*}\right\|
$$

Since $\mathcal{E}_{k} \geq \frac{1}{2}\left\|z_{k}-x^{*}\right\|^{2}$, we deduce that

$$
\left\|z_{k}-x^{*}\right\|^{2} \leq 2 \mathcal{E}_{1}+\sum_{j=1}^{k} 2\left(1+\theta_{j}\right)\left\|r_{j-1}\right\| \times\left\|z_{j}-x^{*}\right\| .
$$

Let us apply the Gronwall lemma A.4 with $a_{j}=\left\|z_{j}-x^{*}\right\|, b_{j}=2\left(1+\theta_{j}\right)\left\|r_{j-1}\right\|$, and $c=\sqrt{2 \mathcal{E}_{1}}$. We obtain

$$
\left\|z_{k}-x^{*}\right\| \leq \sqrt{2 \mathcal{E}_{1}}+\sum_{j=1}^{\infty} 2\left(1+\theta_{j}\right)\left\|r_{j-1}\right\| .
$$

By assumption (74), $\sqrt{2 \mathcal{E}_{1}}+\sum_{j=1}^{\infty} 2\left(1+\theta_{j}\right)\left\|r_{j-1}\right\|$ is a positive finite real number. Returning to (86) we obtain

$$
\mathcal{E}_{k} \leq C:=\mathcal{E}_{1}+\sum_{j=2}^{k}\left(1+\theta_{j}\right)\left\|r_{j-1}\right\|\left(\sqrt{2 \mathcal{E}_{1}}+\sum_{j=1}^{\infty} 2\left(1+\theta_{j}\right)\left\|r_{j-1}\right\|\right) .
$$

By definition of $\mathcal{E}_{k}$, we obtain, for all $k \geq k_{1}$

$$
\frac{\lambda_{k-1}}{g_{k-1}}\left(1+\theta_{k}\right)\left(\Phi\left(x_{k}\right)-\Phi\left(x^{*}\right)\right) \leq \mathcal{E}_{k} \leq C,
$$

which gives the claim. The last item follows directly by summing (84).

Remark 8.2. According to the relations between the parameters, the summability assumption on the errors can be equivalently formulated in terms of $e_{k}$ as

$$
\sum_{k \geq 1} \frac{1}{g_{k}}\left\|e_{k}\right\|<+\infty
$$

When $\theta=1$ and $\alpha_{k}=1-\frac{\alpha}{k}$, using $\frac{1}{g_{k}}=t_{k+1}$, we recover the condition $\sum_{k \geq 1} k\left\|e_{k}\right\|<+\infty$ considered in [8]. 
Remark 8.3. Because of its numerical importance, several papers have been devoted to the study of perturbation, errors in accelerated proximal-gradient methods. One can consult Aujol-Dossal [13], Schmidt-Le Roux-Bach [34], and Villa-Salzo-Baldassarres-Verri [35].

\section{Appendix A. Some AuXiLiary Results}

The following auxiliary lemmas are used throughout the paper. To establish the weak convergence of the iterates of (IP) $\alpha_{k}, \beta_{k}$, we apply Opial's Lemma [27], that we recall in its discrete form.

Lemma A.1. Let $S$ be a nonempty subset of $\mathcal{H}$, and $\left(x_{k}\right)$ a sequence in $\mathcal{H}$. Assume that

(i) every sequential weak cluster point of $\left(x_{k}\right)$, as $k \rightarrow+\infty$, belongs to $S$;

(ii) for every $z \in S, \lim _{k \rightarrow+\infty}\left\|x_{k}-z\right\|$ exists.

Then $\left(x_{k}\right)$ converges weakly as $k \rightarrow+\infty$ to a point in $S$.

Owing to the next lemma, we are able to estimate the rate of convergence of a sequence $\left(\varepsilon_{k}\right)$ supposed to be non-increasing and summable with respect to weight coefficients, see [5, Lemma 21] for the proof.

Lemma A.2. Let $\left(\tau_{k}\right)$ be a nonnegative sequence such that $\sum_{k=1}^{+\infty} \tau_{k}=+\infty$. Assume that $\left(\varepsilon_{k}\right)$ is a non-negative and non-increasing sequence satisfying $\sum_{k=1}^{+\infty} \tau_{k} \varepsilon_{k}<+\infty$. Then we have

$$
\varepsilon_{k}=o\left(\frac{1}{\sum_{i=1}^{k} \tau_{i}}\right) \quad \text { as } k \rightarrow+\infty .
$$

The following result shows the summability of a sequence $\left(a_{k}\right)$ satisfying some suitable inequality.

Lemma A.3. Given a non-negative sequence $\left(\alpha_{k}\right)$ satisfying $\left(K_{0}\right)$, let $\left(t_{k}\right)$ be the sequence defined by $t_{k}=$ $1+\sum_{i=k}^{+\infty} \prod_{j=k}^{i} \alpha_{j}$. Let $\left(a_{k}\right)$ and $\left(\omega_{k}\right)$ be two sequences of nonnegative numbers such that

$$
a_{k+1} \leq \alpha_{k} a_{k}+\omega_{k},
$$

for all $k \geq 0$. If $\sum_{k=0}^{+\infty} t_{k+1} \omega_{k}<+\infty$, then $\sum_{k=0}^{+\infty} a_{k}<+\infty$.

Proof. By Lemma 2.1, we have $t_{k+1} \alpha_{k}=t_{k}-1$. Multiplying inequality (89) by $t_{k+1}$ gives

$$
t_{k+1} a_{k+1} \leq\left(t_{k}-1\right) a_{k}+t_{k+1} \omega_{k},
$$

or equivalently $a_{k} \leq\left(t_{k} a_{k}-t_{k+1} a_{k+1}\right)+t_{k+1} \omega_{k}$. By summing from $k=0$ to $n$, we obtain

$$
\begin{aligned}
\sum_{k=0}^{n} a_{k} & \leq t_{0} a_{0}-t_{n+1} a_{n+1}+\sum_{k=0}^{n} t_{k+1} \omega_{k} \\
& \leq t_{0} a_{0}+\sum_{k=0}^{+\infty} t_{k+1} \omega_{k}<+\infty \quad \text { by assumption. }
\end{aligned}
$$

The conclusion follows by letting $n$ tend to $+\infty$.

Lemma A.4 ([8, Lemma 5.14]). Let $\left(a_{k}\right)$ be a sequence of nonnegative numbers such that, for all $k \in \mathbb{N}$, $a_{k}^{2} \leq c^{2}+\sum_{j=1}^{k} b_{j} a_{j}$, where $\left(b_{j}\right)$ is a summable sequence of nonnegative numbers, and $c \geq 0$. Then, for all $k \in \mathbb{N}, a_{k} \leq c+\sum_{j=1}^{\infty} b_{j}$.

\section{REFERENCES}

[1] S. Adly, H. Атtouch, A. Савот, Finite time stabilization of nonlinear oscillators subject to dry friction, Nonsmooth Mechanics and Analysis, Adv. Mech. Math., 12 (2006), Springer, New York, pp. 289-304.

[2] F. Álvarez, H. Aтtouch, J. Bolte, P. Redont, A second-order gradient-like dissipative dynamical system with Hessiandriven damping. Application to optimization and mechanics, J. Math. Pures Appl., 81 (2002), No. 8, pp. 747-779.

[3] V. Apidopoulos, J.-F. Aujol, CH. Dossal, Convergence rate of inertial Forward-Backward algorithm beyond Nesterov's rule, HAL-01551873, (2017), to appear in Mathematical Programming.

[4] H. Аттоuch, А. САвот, Asymptotic stabilization of inertial gradient dynamics with time-dependent viscosity, J. Differential Equations, 263 (2017), pp. 5412-5458.

[5] H. Аттоuch, A. Савот, Convergence rates of inertial forward-backward algorithms, SIAM J. Optim., 28 (1) (2018), pp. 849-874.

[6] H. Aтtouch, A. Савот, Z. Chbani, H. Riahi, Accelerated forward-backward algorithms with perturbations. Application to Tikhonov regularization, Journal of Optimization Theory and Applications, 179 (2018), pp. 1-36.

[7] H. Attouch, Z. Chbani, H. Riahi, Fast proximal methods via time scaling of damped inertial dynamics, HAL-01939292, 2018.

[8] H. Attouch, Z. Chbani, J. Peypouquet, P. Redont, Fast convergence of inertial dynamics and algorithms with asymptotic vanishing viscosity, Math. Program. Ser. B 168 (2018), pp. 123-175.

[9] H. Aтtouch, Z. Chbani, H. Riahi, Rate of convergence of the Nesterov accelerated gradient method in the subcritical case $\alpha \leq 3$, arXiv:1706.05671v1 [math.OC] 2017, to appear in ESAIM-COCV. 
[10] H. Attouch, P.E. Maingé, P. Redont, A second-order differential system with Hessian-driven damping; Application to non-elastic shock laws, Differential Equations and Applications, 4 (2012), No. 1, pp. 27-65.

[11] H. Aтtouch, J. Peypouquet, The rate of convergence of Nesterov's accelerated forward-backward method is actually faster than $1 / k^{2}$, SIAM J. Optim., 26 (2016), No. 3, pp. 1824-1834.

[12] H. Аtтouch, J. Peypouquet, P. Redont, Fast convex minimization via inertial dynamics with Hessian driven damping, J. Differential Equations, 261 , Issue 10, (2016), pp. 5734-5783.

[13] J.-F. Aujol, Ch. Dossal, Stability of over-relaxations for the Forward-Backward algorithm, application to FISTA, SIAM J. Optim., 25 (2015), No. 4, pp. 2408-2433.

[14] J.-F. Aujol, Ch. Dossal, Optimal rate of convergence of an ODE associated to the Fast Gradient Descent schemes for $b>0$, 2017, https://hal.inria.fr/hal-01547251v2.

[15] H. Bauschke, P. L. Combettes, Convex Analysis and Monotone Operator Theory in Hilbert spaces, CMS Books in Mathematics, Springer, (2011).

[16] A. Beck, M. Teboulle, A fast iterative shrinkage-thresholding algorithm for linear inverse problems, SIAM J. Imaging Sci., 2 (2009), No. 1, pp. 183-202.

[17] R. I. Bot, E. R. Csetnek, S.C. LÁszló, A second order dynamical approach with variable damping to nonconvex smooth minimization, to appear in Applicable Analysis, (2018).

[18] A. Chambolle, Ch. Dossal, On the convergence of the iterates of the Fast Iterative Shrinkage Thresholding Algorithm, Journal of Optimization Theory and Applications, 166 (2015), pp. 968-982.

[19] P.L. Combettes, V.R. WAJs, Signal recovery by proximal forward-backward splitting, Multiscale Model. Simul., 4 (2005), pp. $1168-1200$.

[20] O. GüLER, On the convergence of the proximal point algorithm for convex optimization, SIAM J. Control Optim., 29 (1991), pp. 403-419.

[21] O. GüLER, New proximal point algorithms for convex minimization, SIAM Journal on Optimization, 2 (1992), No. 4, pp. 649-664.

[22] D. Kim, J.A. Fessler, Optimized first-order methods for smooth convex minimization, Math. Program. 159 (2016), No. 1, pp. 81-107.

[23] J. Liang, J. FAdili, G. Peyré, Local linear convergence of forward-backward under partial smoothness, Advances in Neural Information Processing Systems, 2014, pp. 1970-1978.

[24] D.A. Lorenz, Th. Pock, An inertial forward-backward algorithm for monotone inclusions, J. Math. Imaging and Vision, 51 (2015), pp. 311-325.

[25] R. MAY, Asymptotic for a second-order evolution equation with convex potential and vanishing damping term, Turkish Journal of Mathematics, 41 (2017), No. 3, pp. 681-685.

[26] Y. Nesterov, A method of solving a convex programming problem with convergence rate O(1/k2), Soviet Mathematics Doklady, 27 (1983), pp. 372-376.

[27] Z. OpIAL, Weak convergence of the sequence of successive approximations for nonexpansive mappings, Bull. Amer. Math. Soc., 73 (1967), pp. 591-597.

[28] N. Parikh, S. Boyd, Proximal algorithms, Foundations and trends in optimization, volume 1, (2013), pp. $123-231$.

[29] J. Peypouquet, Convex optimization in normed spaces: theory, methods and examples. Springer, 2015.

[30] J. Peypouquet, S. Sorin, Evolution equations for maximal monotone operators: asymptotic analysis in continuous and discrete time, J. Convex Anal, 17 (2010), No. 3-4, pp. 1113-1163.

[31] B.T. PolyaK, Some methods of speeding up the convergence of iteration methods, U.S.S.R. Comput. Math. Math. Phys., 4 (1964), pp. 1-17.

[32] B.T. POLYAK, Introduction to optimization. New York: Optimization Software. (1987)

[33] R.T. Rockafellar, Monotone operators and the proximal point algorithm, SIAM J. Control Optim., 14 (1976), No. 5, pp. $877-898$.

[34] M. Schmidt, N. Le Roux, F. BACH, Convergence rates of inexact proximal-gradient methods for convex optimization, NIPS'11 - 25 th Annual Conference on Neural Information Processing Systems, Dec 2011, Grenada, Spain. (2011) HAL inria-00618152v3.

[35] S. Villa, S. Salzo, L. Baldassarres, A. Verri, Accelerated and inexact forward-backward, SiAM J. Optim., 23 (2013), No. 3, pp. 1607-1633.

[36] B. Shi, S. S. Du, M. I. Jordan, W. J. Su, Understanding the acceleration phenomenon via high-resolution differential equations, arXiv:submit/2440124[cs.LG] 21 Oct 2018.

[37] W. J. Su, S. Boyd, E. J. CANDÈs, A differential equation for modeling Nesterov's accelerated gradient method: theory and insights. Neural Information Processing Systems 27 (2014), pp. 2510-2518.

imaG, Univ. Montpellier, CNRS, Montpellier, France

Email address: hedy.attouch@umontpellier.fr

Cadi Ayyad university, Faculty of Sciences Semlalia, Mathematics, 40000 Marrakech, Morroco

Email address: chbaniz@uca.ac.ma

Cadi Ayyad university, Faculty of Sciences Semlalia, Mathematics, 40000 Marrakech, Morroco

Email address: h-riahi@uca.ac.ma 